\title{
PLURIPOLAR HULLS AND CONVERGENCE SETS
}

\author{
A Dissertation by \\ Juan Chen \\ Master of Science, Tianjin University of Technology and Education, 2012 \\ Bachelor of Science, Tianjin University of Technology and Education, 2009
}

Submitted to the Department of Mathematics, Statistics and Physics and the faculty of the Graduate School of

Wichita State University

in partial fulfillment of

the requirements for the degree of

Doctor of Philosophy

May 2018 
(C) Copyright 2018 by Juan Chen

All Rights Reserved 


\section{PLURIPOLAR HULLS AND CONVERGENCE SETS}

The following faculty members have examined the final copy of this dissertation for form and content, and recommend that it be accepted in partial fulfillment of the requirement for the degree of Doctor of Philosophy with a major in Applied Mathematics.

Daowei Ma, Committee Chair

Thomas K. Delillo, Committee Member

Buma L. Fridman, Committee Member

Lop-Hing Ho, Committee Member

Chengzong Pang, Committee Member

Accepted for the College of Liberal Arts and Sciences

Ron Matson, Dean

Accepted for the Graduate School

Dennis Livesay, Dean 


\section{DEDICATION}

To my family 


\section{ACKNOWLEDGEMENTS}

This dissertation would not have been possible without the guidance and help of several individuals who in one way or another contributed and extended their valuable assistance in the preparation and completion of this study. First of all I would like to express my gratitude to my advisor Professor Daowei Ma, who was truly an inspiration for providing me with ideas and thoughts. He has given me invaluable advice and suggestions throughout the work with this dissertation. Without his guidance, this dissertation would not be possible. Secondly, I would like to take this opportunity to thank Professor Buma Fridman, Professor Lop-Hing Ho, and my classmate Xin Wei, who were in the complex analysis seminar which was instrumental towards the completion of this dissertation. Also I would like to thank the graduate coordinator Professor Ziqi Sun, who has been kind to all graduate students, and all professors in the Mathematics Department at Wichita State University, who have taught me and who have provided their help. Finally, I would like to say thanks to my friends and colleagues with whom I have exchanged mathematics thoughts during my doctorial program. Last but not the least, I would like to thank my husband Xiaodong Xing for his patience and support, and my parents for their understanding in the period when I prepared this dissertation. 


\begin{abstract}
The pluripolar hull of a pluripolar set $\mathrm{E}$ in $\mathbb{P}^{n}$ is the intersection of all complete pluripolar sets in $\mathbb{P}^{n}$ that contain $E$. We prove that the pluripolar hull of each compact pluripolar set in $\mathbb{P}^{n}$ is $F_{\sigma}$. The convergence set of a divergent formal power series $f\left(z_{0}, \ldots, z_{n}\right)$ is the set of all "directions" $\xi \in \mathbb{P}^{n}$ along which $f$ is convergent. We prove that the union of the pluripolar hulls of a countable collection of compact pluripolar sets in $\mathbb{P}^{n}$ is the convergence set of some divergent series $f$, which is more general than the result of Ma and Neelon (J. Complex Analysis and its Synergies 1:4 2015). The convergence sets on $\Gamma:=\{[1: z: \psi(z)]: z \in \mathbb{C}\} \subset \mathbb{C}^{2} \subset \mathbb{P}^{2}$, where $\psi$ is a transcendental entire holomorphic function, are also studied and we obtain that a subset on $\Gamma$ is a convergence set in $\mathbb{P}^{2}$ if and only if it is a countable union of compact projectively convex sets and that the union of a countable collection of convergence sets on $\Gamma$ is a convergence set.
\end{abstract}




\section{TABLE OF CONTENTS}

\section{Chapter}

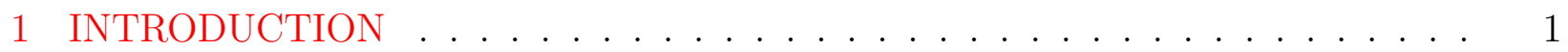

2 THE DEFINITIONS AND CERTAIN PROPERTIES . . . . . . . . . . . . . . . 6

2.1 Plurisubharmonic functions and $\omega$-plurisubharmonic functions . . . . . . . . 6

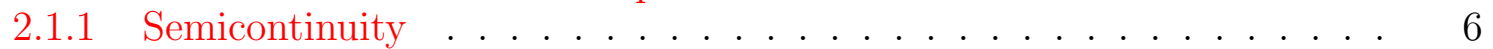

2.1.2 Plurisubharmonic functions . . . . . . . . . . . . . 7

$2.1 .3 \omega$-plurisubharmonic functions . . . . . . . . . . . . . . . 9

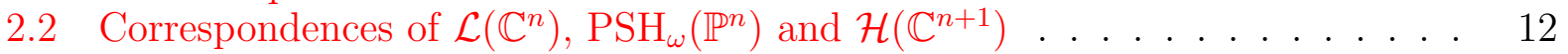

2.3 Pluripolar sets . . . . . . . . . . . . . . . . . . . 16

2.3.1 Pluripolar sets in $\mathbb{C}^{n} \ldots \ldots \ldots \ldots$. . . . . . . . . . . . . . . . . .

2.3.2 Pluripolar sets in $\mathbb{P}^{n} \ldots \ldots \ldots$. . . . . . . . . . . . . . 18

2.4 Pluripolar hulls in $\mathbb{P}^{n} \ldots \ldots$. . . . . . . . . . . . . . . . . . . . . . . . 19

3 RELATIVE $\omega$-PLURISUBHARMONIC EXTREMAL FUNCTIONS . . . . . . . 21

3.1 Relative $\omega$-Plurisubharmonic Extremal Functions . . . . . . . . . . . . . . 21

3.2 Pluripolar hulls in terms of $\omega$-plurisubharmonic functions . . . . . . . . . . . 30

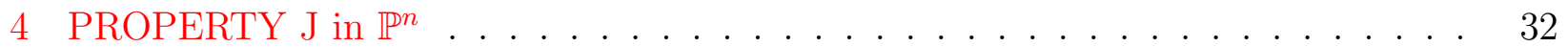

4.1 Property J in $\mathbb{P}^{n} \ldots \ldots \ldots$. . . . . . . . . . . . . . . . . . . 32

4.2 Pluripolar hulls in terms of homogeneous polynomials . . . . . . . . . . 35

5 CONVERGENCE SETS . . . . . . . . . . . . . . . . . . . . 38

5.1 Convergence Sets in $\mathbb{P}^{n} \ldots \ldots \ldots$. . . . . . . . . . . . . . . . 38

5.2 Convergence Sets in $\mathbb{C}^{n} \ldots \ldots \ldots$. . . . . . . . . . . . . 42

6 CONVERGENCE SETS ON $\mathrm{O} \ldots \ldots \ldots \ldots$

6.1 Polynomial Hulls and projective hulls . . . . . . . . . . . . . . . . 45

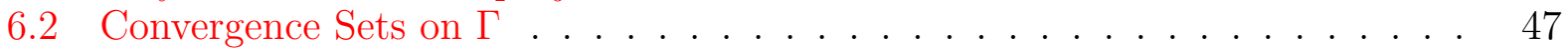

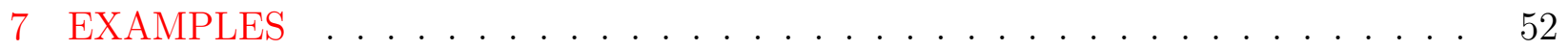

8 CONCLUSION AND FUTURE WORK . . . . . . . . . . . . . . . . 54

REFERENCES . . . . . . . . . . . . . . . . . . . . 55 


\section{CHAPTER 1 \\ INTRODUCTION}

A formal power series $f(z)=f\left(z_{0}, z_{1}, \ldots, z_{n}\right)=\sum_{\alpha} a_{\alpha} z^{\alpha} \in \mathbb{C}\left[\left[z_{0}, z_{1}, \ldots, z_{n}\right]\right]$ with coefficients in $\mathbb{C}$ is said to be convergent if it converges absolutely in a neighborhood of the origin in $\mathbb{C}^{n+1}$. Otherwise we say it diverges. So $f$ converges if and only if there is a number $C>0$ such that $\left|a_{\alpha}\right| \leq C^{|\alpha|+1}$, for each $\alpha \in \mathbb{N}^{n+1}$.

A beautiful classical result of Hartogs (see [12]), which can be interpreted as a formal analog of Hartogs' theorem on separate analyticity, states that a series $f$ converges if and only if $f_{z}(t):=f\left(z_{0} t, z_{1} t \ldots, z_{n} t\right)$, as a series in $t$, converges for all $z \in \mathbb{C}^{n+1}$. But a divergent power series still may converge in some directions, and this engenders a natural and desirable problem of classifying the convergence sets of divergent power series. Since, for $z \neq 0, f_{z}(t)$ converges if and only if $f_{w}(t)$ converges for all $w \in \mathbb{C}^{n+1}$ on the affine line through $z$, we may identify the set of all $z \in \mathbb{C}^{n+1}$ for which $f_{z}$ converges with a subset of $\mathbb{P}^{n}$. The convergence set $\operatorname{Conv}(f)$ of a divergent power series $f$ is defined to be the set of all directions $\xi \in \mathbb{P}^{n}$ such that $f_{z}(t)$ is convergent for some $z \in \pi^{-1}(\xi)$, where $\pi: \mathbb{C}^{n+1} \backslash\{0\} \rightarrow \mathbb{P}^{n}$ is the natural projection. For the case $n=1$, P. Lelong (see [17]) proved that the convergence set of a divergent series $f\left(z_{1}, z_{2}\right)$ is an $F_{\sigma}$ polar set (i.e., a countable union of closed sets of vanishing logarithmic capacity) in $\mathbb{P}^{1}$, and moreover, every $F_{\sigma}$ polar subset of $\mathbb{P}^{1}$ is contained in the convergence set of a divergent series $f\left(z_{1}, z_{2}\right)$. S.S. Abhyankar and T.T. Moh studied convergence sets of divergent power series in [1], where they showed that a convergence set has Lebesgue measure 0. The optimal result was later obtained by A. Sathaye (see [27]) who proved that the class of convergence sets of divergent power series $f\left(z_{1}, z_{2}\right)$ is precisely the class of $F_{\sigma}$ polar sets in $\mathbb{P}^{1}$ which was also proved by J. Ribón (see [24]) with a different method. Levenberg and Molzon obtained in [19] that a convergence set is pluripolar and that a compact complete pluripolar set in $\mathbb{C}^{n}$ is a convergence set in $\mathbb{C}^{n}$. 
Convergence sets of formal power series are applied in many areas of mathematics including complex dynamics and investigation of small divisors. In [22] Pérez-Marco studied polynomial families of dynamical systems presenting problems of small divisors where power series appear naturally and the main result there essentially says that convergence sets in $\mathbb{C}^{n}$ are pluripolar.

In order to study the collection $\operatorname{Conv}\left(\mathbb{P}^{n}\right)$ of convergence sets of divergent series in higher dimensions, the class $\mathrm{PSH}_{\omega}\left(\mathbb{P}^{n}\right)$ of $\omega$-plurisubharmonic functions on $\mathbb{P}^{n}$ with respect to the form $\omega:=d d^{c} \log |Z|$ on $\mathbb{P}^{n}$ was considered in [21], where the authors proved that $\operatorname{Conv}\left(\mathbb{P}^{n}\right)$ contains projective hulls of compact pluripolar sets and countable unions of projective varieties, and showed that each convergence set (of divergent power series) is a countable union of projective hulls of compact pluripolar sets. Their main result states that a countable union of closed complete pluripolar sets in $\mathbb{P}^{n}$ belongs to $\operatorname{Conv}\left(\mathbb{P}^{n}\right)$, which generalized the results of P. Lelong (see [17]), Levenberg and Molzon (see [19]), and Sathaye (see [27]). Convergence sets of formal power series $f(z, t)=\sum_{n=0}^{\infty} f_{n}(z) t^{n}$ with $f_{n}(z)$ holomorphic on a domain $\Omega \subset \mathbb{C}$ was studied in [2], where the authors obtained a characterization of the convergence sets in $\Omega$, which says that a subset of $\mathbb{C}$ is a convergence set if and only if it is $\sigma$-convex. We get some idea of [2] to study convergence sets on $\Gamma$ (see Chapter 6).

In this paper, we consider the class $\operatorname{PSH}_{\omega}\left(\mathbb{P}^{n}\right)$ where $\omega$ is the form mentioned above, introduce relative $\omega$-plurisubharmonic extremal functions and Property $\mathrm{J}$ in $\mathbb{P}^{n}$, and establish some results on pluripolar hulls and convergence sets. The main result of this paper generalizes the main result of [21].

Theorem 5.1.5 Let $\left\{K_{j}\right\}$ be a sequence of compact pluripolar sets in $\mathbb{P}^{n}$. Then

$$
K:=\cup_{j=1}^{\infty} K_{j}^{*}
$$

is a convergence set.

In other words, we prove that $\cup_{j=1}^{\infty} K_{j}^{*}$ is a convergence set, where each $K_{j}^{*}$ is the pluripolar hull of the compact pluripolar set $K_{j}$. Of course this is more general than the main result 
in [21], because $K_{j}^{*}$ in general is neither compact nor a complete pluripolar set. In order to prove the main theorem, we introduce the notion of relative $\omega$-plurisubharmonic extremal functions. We use the extremal functions to decompose the pluripolar hull $K_{j}^{*}$ into "sublevel sets" $K_{j}^{(m)}$, and then construct $E_{k}:=\cup_{j=1}^{k} K_{j}^{(k)}$ to rewrite $\cup_{j=1}^{\infty} K_{j}^{*}$ as a union $\cup_{k=1}^{\infty} E_{k}$. Unfortunately, in general $E_{k}$ is not a complete pluripolar set, and $E_{k}$ does not have Property $\mathrm{J}$ (i.e., $E_{k}$ does not have Property $\mathrm{J}$ with respect to every point in $\mathbb{P}^{n} \backslash E_{k}$ ), though $E_{k}$ does have Property J with respect to each $X \in \mathbb{P}^{n} \backslash \cup_{j=1}^{\infty} K_{j}^{*}$. Hence with some additional efforts we are able to modify parts of the reasoning in [21] to finish the proof here (see Section 5.1 in Chapter 5).

Let $D$ be an open set in $\mathbb{C}^{n}$ and let $F$ be a pluripolar set in $D$. A well-known result from Josefson (see [15]) is that there exists a plurisubharmonic function $u$ on $\mathbb{C}^{n}$ such that $F \subset\{u=-\infty\}$. If $F$ is not a complete pluripolar set, then it may happen that each plurisubharmonic function which is $-\infty$ on $F$ is $-\infty$ on a larger set. In order to study how a pluripolar subset $F \subset D$ propagates, the notion of the pluripolar hull was introduced. The pluripolar hull $F_{D}^{*}$ of a pluripolar set $F \subset D$ with respect to $D$ is defined to be the intersection of all complete pluripolar sets in $D$ that contain $F$, that is, $F_{D}^{*}=\cap\left\{z \in \mathbb{C}^{n}: u(z)=-\infty\right\}$ where the intersection is taken over all plurisubharmonic functions $u$ on $D$ that are $-\infty$ on F. In [3], E. Berdford and B.A. Taylor considered pluripolar hulls of pluripolar sets with respect to $\mathbb{C}^{n}$ and obtained that a smooth algebraic hypersurface $H$ in $\mathbb{P}^{n}$ is not a subset of the pluripolar hull of a pluripolar set $F \subset \mathbb{C}^{n}$ with $F \cap H=\varnothing$. It follows from a result of Zeriahi (see [32]) that if the pluripolar hull $F_{D}^{*}$ of a pluripolar subset $F$ of a pseudoconvex domain $D$ is both $F_{\sigma}$ and $G_{\delta}$, then $F_{D}^{*}$ is a complete pluripolar set in $D$. The negative pluripolar hull $F_{D}^{-}$was later introduced by Levenberg and Poletsky in [20] where they characterized the negative pluripolar hull $F_{D}^{-}$by using the plurisubharmonic measure and eatablished a connection between the pluripolar hull $F_{D}^{*}$ and the negative pluripolar hull $F_{D}^{-}$. They also provided a number of techniques, which were applied by A. Ediganian and J. Wiegerinck, T. Edlund and B. Jöricks and so on (see [7, 8, 9] and the references therein), 
to study pluripolar hulls of graphs of some holomorphic functions. In [21], D. Ma and T. Neelon introduced the pluripolar hull $E^{*}$ of a pluripolar subset $E$ in $\mathbb{P}^{n}$ and showed that the pluripolar hull $K^{*}$ of a compact pluripolar set $K$ in $\mathbb{P}^{n}$ contains the projective hull $\hat{K}$ of $K$. In this dissertation, the pluripolar hull of a pluripolar set in $\mathbb{P}^{n}$ is considered. We characterize the pluripolar hull of a pluripolar set in $\mathbb{P}^{n}$ in terms of relative $\omega$-plurisubharmonic extremal functions and in terms of homogeneous polynomials, and obtain that the pluripolar hull of each compact pluripolar set in $\mathbb{P}^{n}$ is $F_{\sigma}$, which generalizes the result of Levenberg and Poletsky (see [20]).

In Chapter 2, we give some necessary background in pluripotential theory. Section 2.1 contains semicontinuity, plurisubharmonic functions and $\omega$-plurisubharmonic functions, and some of their properties which we shall need later. In addition, the correspondences of $\mathcal{L}\left(\mathbb{C}^{n}\right), \operatorname{PSH}_{\omega}\left(\mathbb{P}^{n}\right)$ and $\mathcal{H}\left(\mathbb{C}^{n+1}\right)$ are given in Section 2.1. It contains pluripolar sets in $\mathbb{C}^{n}$ of pluripotential theory in Section 2.2, and pluripolar sets in $\mathbb{P}^{n}$ were introduced in terms of $\omega$-plurisubharmonic functions there. In Section 2.3 we introduce pluripolar hulls in $\mathbb{P}^{n}$ and study their properties.

In Chapter 3, we introduce the notion of relative $\omega$-plurisubharmonic extremal functions in $\mathbb{P}^{n}$ in terms of $\omega$-plurisubharmonic functions, give an explicit example of the relative $\omega$ plurisubharmonic extremal function (see Example 3.1), and gather some useful properties of them in Section 3.1 and 3.2. Section 3.3 provides a characterization of the pluripolar hull of a pluripolar set in $\mathbb{P}^{n}$ by using relative $\omega$-plurisubharmonic extremal functions (see Theorem 3.2.1 and Theorem 3.2.3).

Property $J$ in $\mathbb{P}^{n}$ is defined by means of homogeneous polynomials in Chapter 4 . In Section 4.1, we establish a useful formula (see Proposition 4.1.3)

$$
\sigma(Z, s, K)=\log Q_{K, Z}\left(e^{-s}\right),
$$

which is motivated by [16, Theorem 5.1.6(i)] and [31, Proposition 2.10]. Then in Section 4.2 , because of the above formula we are able to give an interpretation of the pluripolar hull of a pluripolar set in $\mathbb{P}^{n}$ in terms of homogeneous polynomials. Hence we obtain that 
the pluripolar hull of each compact pluripolar set in $\mathbb{P}^{n}$ is $F_{\sigma}$ (see Theorem 4.2.2). We further establish the connection of Property J, a complete pluripolar set and relative $\omega$ plurisubharmonic extremal functions (see Theorem 4.2.3).

Convergence sets are studied in Chapter 5. We first study convergence sets in $\mathbb{P}^{n}$ and prove the main result of this paper in Section 5.1. In Section 5.2, we consider the formal power series of the type

$$
g(t, z)=\sum_{k=0}^{\infty} P_{k}(z) t^{k} \in \mathbb{C}\left[z_{1}, z_{2}, \ldots, z_{n}\right][[t]],
$$

where $P_{k}$ are polynomials with $\operatorname{deg} P_{k} \leq k$. The convergence set $\operatorname{Conv}(g)$ of a divergent power series $g(t, z)$ is defined to be the set of $z \in \mathbb{C}^{n}$ for which $g(t, z)$ converges as a series of $t$, which was also considered in [21] with the result that a countable union of closed complete pluripolar sets is a convergence set in $\mathbb{C}^{n}$. We then study convergence sets in $\mathbb{C}^{n}$ and obtain that a countable union of the pluripolar hulls of closed pluripolar sets in $\mathbb{C}^{n}$ is a convergence set in $\mathbb{C}^{n}$ (see Theorem 5.2.4).

In Chapter 6, we introduce polynomial hulls, projective hulls and study some properties of them in Section 6.1. Convergence sets on $\Gamma:=\{[1: z: \psi(z)]: z \in \mathbb{C}\} \subset \mathbb{C}^{2} \subset \mathbb{P}^{2}$ with a transcendental entire holomorphic function $\psi$ are considered in Section 6.2, where we prove that a subset on $\Gamma$ is a convergence set in $\mathbb{P}^{n}$ if and only if it is a countable union of compact projectively convex sets (see Theorem 6.2.4), and hence we conclude that the union of a countable collection of convergence sets on $\Gamma$ is a convergence set on $\Gamma$ (see Corollary 6.2.5).

We give some examples of convergence sets or non-convergence sets in Chapter 7.

For the convenience of the reader we provide a table of notation at the end. 


\section{CHAPTER 2}

\section{THE DEFINITIONS AND CERTAIN PROPERTIES}

\subsection{Plurisubharmonic functions and $\omega$-plurisubharmonic functions}

\subsubsection{Semicontinuity}

As part of the definition of plurisubharmonic functions and $\omega$-plurisubharmonic functions, we have to take a brief look at upper semicontinuous functions.

Definition 2.1.1. Let $\mathcal{X}$ be a metric space. A function $u: \mathcal{X} \rightarrow[-\infty, \infty)$ is said to be upper semicontinuous if for each $a \in \mathbb{R}$ the set $\{x \in \mathcal{X}: u(x)<a\}$ is open in $\mathcal{X}$. A function $u$ is said to be lower semicontinuous if $-u$ is upper semicontinuous.

Note that by the above definition $u$ is upper semicontinuous if and only if at each point $x_{0} \in \mathcal{X}$ we have $\limsup _{x \rightarrow x_{0}} u(x)=u\left(x_{0}\right)$, where

$$
\limsup _{x \rightarrow x_{0}} u(x)=\inf _{\varepsilon>0}\left(\sup \left\{u(y): y \in B\left(x_{0}, \varepsilon\right)\right\}\right) .
$$

Informally, an upper (respectively, lower) semicontinuous function can only "jump up" (respectively, "jump down"). For example, the characteristic function of a subset $S \subset \mathcal{X}$ is upper semicontinuous if and only if $S$ is closed in $\mathcal{X}$. Also, $u$ is continuous if and only if $u$ is both upper and lower semicontinuous.

The following Proposition states some properties of upper semicontinuous functions (see e.g., $[16,23])$.

Proposition 2.1.2. Let $K$ be a compact metric space, and let $u: K \rightarrow[-\infty, \infty)$ be an upper semicontinuous function on $K$. Then

(i) $u$ is bounded above on $K$ and attains its bound;

(ii) there exists a sequence $u_{1} \geq u_{2} \geq \cdots$ of continuous functions on $K$ such that $\lim _{j \rightarrow \infty} u_{j}=u$. 
Definition 2.1.3. Let $\mathcal{Y}$ be a nonempty subset of $\mathcal{X}$ and let $u: \mathcal{Y} \rightarrow[-\infty, \infty)$ be a function which is locally bounded from above near each point of $\overline{\mathcal{Y}}$. We define the upper semicontinuous regularization $u^{*}$ of $u$ by

$$
u^{*}(x)=\limsup _{y \rightarrow x, y \in \mathcal{Y}} u(y)=\inf _{\varepsilon>0}(\sup \{u(y): y \in \bar{B}(x, \varepsilon) \cap \mathcal{Y}\}) \quad x \in \overline{\mathcal{Y}} .
$$

Then $u^{*}: \overline{\mathcal{Y}} \rightarrow[-\infty, \infty)$ is upper semicontinuous and $u^{*} \geq u$ in $\mathcal{Y}$. Moreover, if $v: \overline{\mathcal{Y}} \rightarrow[-\infty, \infty)$ is upper semicontinuous and $u \leq v$ in $\mathcal{Y}$, then $u^{*} \leq v$ in $\overline{\mathcal{Y}}$. In particular, a function $u: \overline{\mathcal{X}} \rightarrow[-\infty, \infty)$ is upper semicontinuous if and only if it coincides with its upper semicontinuous regularization.

\subsubsection{Plurisubharmonic functions}

Let $D$ be an open subset of $\mathbb{C}^{n}$.

Definition 2.1.4. An upper semicontinuous function $u: D \rightarrow[-\infty, \infty)$ is said to be plurisubharmonic if for each $z \in D$ and $w \in \mathbb{C}^{n}$, the function $u(z+\lambda w)$ is subharmonic or identically $-\infty$ on every component of the set $\{\lambda \in \mathbb{C}: z+\lambda w \in D\}$.

Example 2.1. If $f$ is analytic, then $\log |f|$ is plurisubharmonic.

We denote by $\operatorname{PSH}(D)$ the family of plurisubharmonic functions on $D$. An upper semicontinuous function $u$ is called plurisuperharmonic if $-u$ is plurisubharmonic.

Proposition 2.1.5. (see $[14,16])$ Let $D$ and $D^{\prime}$ be open sets in $\mathbb{C}^{n}$ and $\mathbb{C}^{m}$, respectively. If $u \in \operatorname{PSH}(D)$ and $f: D^{\prime} \rightarrow D$ is a holomorphic mapping, then $u \circ f$ is plurisubharmonic in $D^{\prime}$.

This implies that plurisubharmonicity is biholomorphically invariant and hence plurisubharmonic functions can be defined on any complex analytic manifold. In fact, Proposition 2.1.5 can be viewed as the invariance criterion for plurisubharmonic functions.

Plurisubharmonicity can also be characterized as follows. 
Proposition 2.1.6. (see [16]) Let $u$ be upper semicontinuous. Then $u \in \operatorname{PSH}(D)$ if and only if for each $z \in D$ and $w \in \mathbb{C}^{n}$ such that $\{z+\lambda w: \lambda \in \mathbb{C},|\lambda| \leq 1\} \subset D$, we have

$$
u(z) \leq \frac{1}{2 \pi} \int_{0}^{2 \pi} u\left(z+e^{i t} w\right) d t .
$$

Proposition 2.1.7. (see [14, 16]) A function $u \in C^{2}(D)$ is plurisubharmonic if and only if

$$
\sum_{j, k=1}^{n} \frac{\partial^{2} u(z)}{\partial z_{j} \partial \bar{z}_{k}} w_{j} \bar{w}_{k} \geq 0
$$

Remark. Proposition 2.1.6 gives us a convenient characterization of plurisubharmonicity in terms of integral means inequality and Proposition 2.1.7 characterizes plurisubharmonic functions in terms of distributional derivatives. The above two characterizations of plurisubharmonic functions follow directly from the definition of plurisubharmonic functions and their analogues for subharmonic functions. We refer readers to [16, Theorem 2.9.1, 2.9.11] and [14, Theorem 2.6.2] for the details.

We now list some properties of plurisubharmonic functions here without proofs (see [16]). In fact, these properties can be derived directly from the subharmonic case, and either the definition of plurisubharmonic functions or the invariance criterion described in Proposition 2.1.5.

Proposition 2.1.8. Let $D$ be an open subset of $\mathbb{C}^{n}$.

(i) The family $\operatorname{PSH}(D)$ is a convex cone, i.e., if $a, b$ are non-negative numbers and $u, v \in \operatorname{PSH}(D)$, then $a u+b v \in \operatorname{PSH}(D)$.

(ii) If $D$ is connected and $\left\{u_{j}\right\}_{j \in \mathbb{N}} \subset \operatorname{PSH}(D)$ is a decreasing sequence, then $u:=$ $\lim _{j \rightarrow \infty} u_{j} \in \operatorname{PSH}(D)$ or $u=-\infty$.

(iii) If $u: D \rightarrow \mathbb{R}$, and if $\left\{u_{j}\right\}_{j \in \mathbb{N}} \subset \mathrm{PSH}(D)$ converges to $u$ uniformly on compact subsets of $D$, then $u \in \operatorname{PSH}(D)$.

Proposition 2.1.9. Let $\mathcal{U} \subset \operatorname{PSH}(D)$ be a family of functions which is locally bounded above. Then the upper semicontinuous regularization $u^{*}$ of $u(z):=\sup \{v(z): v \in \mathcal{U}\}$ is plurisubharmonic in $D$. 
Let $\mathcal{U}, u$ and $u^{*}$ be as in Proposition 2.1.9. A set of the form

$$
\mathscr{N}=\left\{z \in D: u(z)<u^{*}(z)\right\}
$$

is called negligible. The following result of Bedford and Taylor (see [4, Theorem 7.1]) will be used in this dissertation.

Proposition 2.1.10. (see [4]) Negligible sets are pluripolar.

Proposition 2.1.11. Let $\left\{u_{j}\right\}_{j \in \mathbb{N}} \subset \operatorname{PSH}(D)$ be locally uniformly bounded above in $D \subset \mathbb{C}^{n}$. Suppose that

$$
\limsup _{j \rightarrow \infty} u_{j}(z) \leq M
$$

for each $z \in D$ and some constant $M$. Then for each $\varepsilon>0$ and each compact set $W \subset D$, there exists a natural number $j_{0}$ such that for $j \geq j_{0}$,

$$
\sup _{z \in W} u_{j}(z) \leq M+\varepsilon
$$

Remark. This property is known as the Hartogs Lemma (see [16, Theorem 2.6.4]).

The next property of plurisubharmonic functions is known as the removable singularity theorem (see [16, Theorem 2.9.22]) for plurisubharmonic functions.

Proposition 2.1.12. Let $D$ be an open subset of $\mathbb{C}^{n}$, and let $F$ be a closed subset of $D$ of the form $F=\{z \in D: v(z)=-\infty\}$ for some $v \in \operatorname{PSH}(D)$. If $u \in \operatorname{PSH}(D \backslash F)$ is bounded above, then the function $\tilde{u}$ defined by the formula

$$
\tilde{u}(z)= \begin{cases}u(z) & z \in D \backslash F \\ \lim \sup _{w \rightarrow z, w \notin F} u(w) & z \in F\end{cases}
$$

is plurisubharmonic in $D$.

\subsection{3 $\omega$-plurisubharmonic functions}

It follows from the maximum principle that there are no globally defined non-constant plurisubharmonic functions on $\mathbb{P}^{n}$. However, for a fixed positive closed form $\omega$ of bidegree 
$(1,1)$, the class $\mathrm{PSH}_{\omega}\left(\mathbb{P}^{n}\right)$ of $\omega$-plurisubharmonic functions on $\mathbb{P}^{n}$, to be defined below, serves as a substitute of plurisubharmonic functions. The complete pluripolar sets on $\mathbb{P}^{n}$ can be defined in terms of $\omega$-plurisubharmonic functions.

We fix a Kähler form

$$
\omega:=d d^{c} \log |Z|=\frac{i}{2 \pi} \partial \bar{\partial} \log \left(\left|Z_{0}\right|^{2}+\cdots+\left|Z_{n}\right|^{2}\right)
$$

on $\mathbb{P}^{n}$, where $d^{c}=(i / 2 \pi)(\bar{\partial}-\partial)$. Note that $\omega$ is the Fubini-Study form on $\mathbb{P}^{n}$ (see, e.g., $[10$, p. 30]).

Definition 2.1.13. Let $\Omega \subset \mathbb{P}^{n}$ be an open subset of $\mathbb{P}^{n}$. A function $\varphi: \Omega \rightarrow \mathbb{R} \cup\{-\infty\}$ is said to be $\omega$-plurisubharmonic if $\varphi+\psi$ is plurisubharmonic on $U$ for each open set $U \subset \Omega$ and each $C^{\infty}$ function $\psi$ on $U$ with $d d^{c} \psi=\omega$.

For convenience the function $\varphi \equiv-\infty$ is considered to be both plurisubharmonic and $\omega$-plurisubharmonic.

Remark. (a) Equivalently, an upper semicontinuous function $\varphi$ from $\Omega$ to $\mathbb{R} \cup\{-\infty\}$ is called $\omega$-plurisubharmonic if $d d^{c} \varphi+\omega \geq 0$ (see, e.g., [11]).

(b) Observe that $\varphi: \Omega \rightarrow \mathbb{R} \cup\{-\infty\}$ is $\omega$-plurisubharmonic if for each open set $U \subset \Omega$ on which there is a $C^{\infty}$ function $\psi$ with $d d^{c} \psi=\omega$ the function $\varphi+\psi$ is plurisubharmonic on $U$. To see this, let us assume that $\psi, \psi_{1} \in C^{\infty}(U)$ satisfy $d d^{c} \psi=\omega$ and $d d^{c} \psi_{1}=\omega$ and that $\varphi+\psi$ is plurisubharmonic on $U$. Then $d d^{c}\left(\psi_{1}-\psi\right)=0$ and hence $\psi_{1}-\psi$ is pluriharmonic on $U$. It follows that $\varphi+\psi_{1}=(\varphi+\psi)+\left(\psi_{1}-\psi\right)$ is plurisubharmonic.

Denote by $\operatorname{PSH}_{\omega}(\Omega)$ the family of $\omega$-plurisubharmonic functions on $\Omega$. For a homogeneous polynomial $p$, the function $Z \mapsto \log \left(|p(Z)|^{1 / \operatorname{deg} p} /|Z|\right)$ is a prototypical function in $\operatorname{PSH}_{\omega}\left(\mathbb{P}^{n}\right)$. Suppose that $\ell(Z):=a_{0} Z_{0}+\cdots+a_{n} Z_{n}$ is a linear form and $V$ is an open subset of $U_{\ell}:=\left\{Z \in \mathbb{P}^{n}: \ell(Z) \neq 0\right\}$. Then a function $\varphi$ on $V$ is $\omega$-plurisubharmonic if and only if the function $\varphi(Z)+\log (|Z| /|\ell(Z)|)$ is plurisubharmonic. Moreover, a function $\varphi$ on $\Omega \subset \mathbb{P}^{n}$ belongs to $\operatorname{PSH}_{\omega}(\Omega)$ if and only if the function $\varphi(Z)+\log (|Z| /|\ell(Z)|)$ is plurisubharmonic on $\Omega \cap U_{\ell}$ for each linear form $\ell$. 
Proposition 2.1.14. Let $\mathcal{V} \subset \operatorname{PSH}_{\omega}(\Omega)$ be a family of functions which is locally bounded above. Then the upper semicontinuous regularization $\varphi^{*}$ of $\varphi(z):=\sup \{\psi(z): \psi \in \mathcal{V}\}$ is $\omega$-plurisubharmonic in $\Omega$.

Remark. This is an analogue for Proposition 2.1.9.

We now list without proofs the Hartog's lemma and the removable singularity theorem for $\omega$-plurisubharmonic functions which we shall need and which are immediate consequences of their analogue for plurisubharmonic functions.

Proposition 2.1.15. Let $\left\{\varphi_{j}\right\}_{j \in \mathbb{N}} \subset \operatorname{PSH}_{\omega}(\Omega)$ be locally uniformly bounded above in $\Omega \subset \mathbb{P}^{n}$. Suppose that

$$
\limsup _{j \rightarrow \infty} \varphi_{j}(z) \leq M
$$

for each $z \in \Omega$ and some constant $M$. Then for each $\varepsilon>0$ and each compact set $K \subset \Omega$, there exists a natural number $j_{0}$ such that for $j \geq j_{0}$,

$$
\sup _{z \in K} u_{j}(z) \leq M+\varepsilon
$$

Proposition 2.1.16. Let $\Omega$ be a subset of $\mathbb{P}^{n}$, and let $K$ be a compact subset of $\Omega$ of the form $K=\{z \in \Omega: \psi(z)=-\infty\}$ for some $\psi \in \operatorname{PSH}_{\omega}(\Omega)$. If $\varphi \in \operatorname{PSH}(\Omega \backslash K)$ is bounded above, then the function $\tilde{\varphi}$ defined by the formula

$$
\tilde{\varphi}(z)= \begin{cases}\varphi(z) & z \in \Omega \backslash K \\ \lim \sup _{w \rightarrow z, w \notin K} \varphi(w) & z \in K\end{cases}
$$

is $\omega$-plurisubharmonic in $\Omega$.

Note that we are able to approximate each plurisubharmonic function $u$ by a decreasing sequence $\left\{u_{j}\right\}$ of smooth plurisubharmonic functions with $u_{j} \rightarrow u$ as $j \rightarrow \infty$, where each $u_{j}$ is obtained by forming the convolution of $u$ with standard smoothing kernels and is defined in any fixed relatively compact subdomain of $D$ (see [16, Theorem 2.9.2]). We also have the approximation theorem for $\omega$-plurisubharmonic functions. 
Proposition 2.1.17. For every $\varphi \in \operatorname{PSH}_{\omega}\left(\mathbb{P}^{n}\right)$, there exists a sequence $\left\{\varphi_{j}\right\}$ of smooth $\omega$-plurisubharmonic functions $\varphi_{j} \in \mathrm{PSH}_{\omega}\left(\mathbb{P}^{n}\right) \cap C^{\infty}$ such that $\varphi_{j}$ decreases towards $\varphi$.

For the detailed proof of Proposition 2.1.17 we refer the reader to [11, Theorem 8.1]. Proposition 2.1.17 will be repeatedly used in this dissertation (see Proposition 4.1.3, Theorem 4.2.3).

\subsection{Correspondences of $\mathcal{L}\left(\mathbb{C}^{n}\right), \mathrm{PSH}_{\omega}\left(\mathbb{P}^{n}\right)$ and $\mathcal{H}\left(\mathbb{C}^{n+1}\right)$}

Let $\mathscr{H}\left(\mathbb{C}^{n+1}\right)$ denote the set of all homogeneous polynomials in $n+1$ variables $z_{0}, \ldots, z_{n}$ with complex coefficients. For $k \geq 0$, let $\mathscr{H}_{k}\left(\mathbb{C}^{n+1}\right)$ denote the set of homogeneous polynomials $p \in \mathscr{H}\left(\mathbb{C}^{n+1}\right)$ such that $p(\lambda z)=\lambda^{k} p(z)$ for all $\lambda \in \mathbb{C}$ and $z \in \mathbb{C}^{n+1}$. So each $\mathscr{H}_{k}\left(\mathbb{C}^{n+1}\right)$ is a $\mathbb{C}$-vector space and $\mathscr{H}\left(\mathbb{C}^{n+1}\right)=\cup_{k=0}^{\infty} \mathscr{H}_{k}\left(\mathbb{C}^{n+1}\right)$.

For $k \geq 0$, let $\mathscr{P}_{k}\left(\mathbb{C}^{n}\right)$ denote the family of polynomials of degree at most $k$ in $n$ variables $z_{1}, \ldots, z_{n}$ with complex coefficients in $\mathbb{C}$. So each $\mathscr{P}_{k}\left(\mathbb{C}^{n}\right)=\oplus_{j=0}^{k} \mathscr{H}_{j}\left(\mathbb{C}^{n}\right)$ is a $\mathbb{C}$-vector space and in particular $\mathscr{P}_{0}\left(\mathbb{C}^{n}\right)=\mathscr{H}_{0}\left(\mathbb{C}^{n}\right)=\mathbb{C}$. Denote by $\mathscr{P}\left(\mathbb{C}^{n}\right)$ the family of all complex polynomials of $n$ variables.

Let $\mathcal{L}\left(\mathbb{C}^{n}\right)$ be the family of plurisubharmonic functions in $\mathbb{C}^{n}$ defined by

$$
\mathcal{L}\left(\mathbb{C}^{n}\right):=\left\{u \in \operatorname{PSH}\left(\mathbb{C}^{n}\right): \sup _{z \in \mathbb{C}^{n}}\left(u(z)-(1 / 2) \log \left(1+|z|^{2}\right)\right)<\infty\right\}
$$

For a polynomial $P \in \mathcal{P}_{k}\left(\mathbb{C}^{n}\right)$, where $k>0$, the function $(1 / k) \log |P(z)|$ is a prototypical member of $\mathcal{L}\left(\mathbb{C}^{n}\right)$.

Denote by $\mathcal{H}\left(\mathbb{C}^{n}\right)$ the family of all functions $u \in \operatorname{PSH}\left(\mathbb{C}^{n}\right)$ which are non-negative homogeneous, i.e., which satisfy $u(\lambda z)=|\lambda| u(z)$ for all $\lambda \in \mathbb{C}$ and $z \in \mathbb{C}^{n}$.

Before proceeding to the correspondences of $\mathcal{L}\left(\mathbb{C}^{n}\right), \mathrm{PSH}_{\omega}\left(\mathbb{P}^{n}\right)$ and $\mathcal{H}\left(\mathbb{C}^{n+1}\right)$, we shall describe one of the most useful features of the plurisubharmonic functions with minimal growth at infinity in the next theorem, which essentially states that plurisubharmonic functions can be fully characterized in terms of complex polynomials (see [31, Theorem 5.1.6]; see also [18, Chapter 2]). 
Theorem 2.2.1. Let $h: \mathbb{C}^{n} \rightarrow[0, \infty)$ and $u: \mathbb{C}^{n} \rightarrow[-\infty, \infty)$ be functions such that $h \not \equiv 0$ and $u \not \equiv-\infty$.

(i) If $h \in C\left(\mathbb{C}^{n}\right) \cap \mathcal{H}\left(\mathbb{C}^{n}\right)$ and $h^{-1}(0)=\{0\}$, then

$$
h(z)=\sup \left\{|Q(z)|^{1 / \operatorname{deg} Q}\right\} \quad\left(z \in \mathbb{C}^{n}\right)
$$

where the supremum is taken over all $Q \in \mathscr{H}\left(\mathbb{C}^{n}\right)$ such that $|Q|^{1 / \operatorname{deg} Q} \leq h$ in $\mathbb{C}^{n}$.

(ii) A sufficient and necessary condition for $h$ to belong to $\mathcal{H}\left(\mathbb{C}^{n}\right)$ is that

$$
h=\left(\limsup _{j \rightarrow \infty}\left|Q_{j}\right|^{1 / j}\right)^{*}
$$

for some sequence $\left\{Q_{j} \in \mathscr{H}\left(\mathbb{C}^{n}\right)\right\}$ with $\operatorname{deg} Q_{j} \leq j$. In particular, if $h \in \mathcal{H}\left(\mathbb{C}^{n}\right)$, then $\log h \in \mathcal{L}\left(\mathbb{C}^{n}\right)$.

(iii) The function $u$ belongs to $\mathcal{L}\left(\mathbb{C}^{n}\right)$ if and only if

$$
e^{u}=\left(\limsup _{j \rightarrow \infty}\left|P_{j}\right|^{1 / j}\right)^{*}
$$

for some sequence $\left\{P_{j} \in \mathscr{P}\left(\mathbb{C}^{n}\right)\right\}$ with $\operatorname{deg} P_{j} \leq j$.

Remark. (a) The above theorem is the unique way to establish the relation between polynomials and plurisubharmonic functions.

(b) If $h$ is representable in the form of the above theorem, then $h$ is continuous: it is upper-semicontinuous since $h \in \operatorname{PSH}\left(\mathbb{C}^{n}\right)$ and it is lower-semicontinuous since for $a \in \mathbb{R}$ the set $\left\{z \in \mathbb{C}^{n}: h(z)>a\right\}=\cup_{j}\left\{z \in \mathbb{C}^{n}:\left|Q_{j}(z)\right|>a\right\}$, as the union of open sets, is open.

(c) The above theorem (i) is actually a theorem in $\mathbb{P}^{n}$. And there is no corresponding version in $\mathbb{C}^{n}$. More precisely, the following statement is incorrect: If $u \in \mathcal{L}\left(\mathbb{C}^{n}\right)$ and $u$ is non-vanishing, then $u(z)=\sup \left\{|p(z)|^{1 / k}:|p(z)|^{1 / k} \leq u, \operatorname{deg} p \leq k\right\}$. In fact, the extension of such $u$ to $z_{0}=0$ must be continuous, which actually turns into the $\mathbb{P}^{n}$ version.

(d) The proof of the above theorem depends on complex analysis (see [31, Theorem 5.1.6]; see also [18, Chapter 2]). 
(e) The "G part" of Siciak's Main Approximation Property ([31, p. 16, 2.13]) is stronger than the usual approximation theorem. The " $H$ part" is equivalent to the usual approximation theorem, because if $\psi_{\varepsilon} \in H \cap C^{\infty}$ then $\psi_{\varepsilon}(z)+\varepsilon|z| \in H_{0}$. Its proof is incorrect: since $\psi_{\lambda}(0, x) \rightarrow 0$ as $\lambda \rightarrow 0$, and since $h(0, x) \neq 0$ in general, it cannot be true that $\psi_{\lambda} \rightarrow h$.

(f) Such function $h$ in (i) does not necessarily satisfy $h^{-1}(0)=\{0\}$. For example, let $h=\sup \left\{\left|Q_{j}\right|^{1 / \operatorname{deg} Q_{j}}\right\}$, where $Q_{j}(z)=\left|z_{2}-z_{1}\right|$ for each $j$. Then $h \in \mathcal{H}\left(\mathbb{C}^{n}\right)$, but $h^{-1}=\left\{z=\left(z_{1}, z_{2}, \ldots, z_{n}\right): z_{1}=z_{2}\right\}$.

(g) If the condition $h^{-1}(0)=\{0\}$ is removed, part (i) of the above theorem fails: consider $u(z)=\sum_{j=1}^{\infty} 2^{-j} \log |z-1 / j| \in \mathcal{L}(\mathbb{C})$ for $z \in \mathbb{C}$. Then $h\left(z_{0}, z_{1}\right)=\left|z_{0}\right| \exp u\left(z_{1} / z_{0}\right)=$ $\Pi_{j=1}^{\infty}\left(z_{1}-z_{0} / j\right)^{2^{-j}} \in \mathcal{H}\left(\mathbb{C}^{2}\right)$, and $h^{-1}(0)=\left\{\left(z_{0}, z_{1}\right) \in \mathbb{C}^{2}: z_{1}=z_{0} / j\right.$ for each $\left.j\right\}$. If there is a family of $Q \in \mathscr{H}\left(\mathbb{C}^{2}\right)$ with $|Q|^{1 / \operatorname{deg} Q} \leq h$ such that $h=\sup \left\{|Q|^{1 / \operatorname{deg} Q}\right\}$, then for each $z_{0} \neq 0,\left|Q\left(z_{0}, z_{0} / j\right)\right|^{1 / \operatorname{deg} Q} \leq h\left(z_{0}, z_{0} / j\right)=0$ implies that $Q \equiv 0$ (since it is impossible that $Q$ has infinitely many zeros) and hence $h \equiv 0$ which is a contradiction.

We now proceed to establish the one-to-one correspondences between $\operatorname{PSH}_{\omega}\left(\mathbb{P}^{n}\right)$ and $\mathcal{L}\left(\mathbb{C}^{n}\right)$ (see Proposition 2.2.2), and between $\mathcal{H}\left(\mathbb{C}^{n+1}\right)$ and $\mathcal{L}\left(\mathbb{C}^{n}\right)$ (see Proposition 2.2.3), respectively, which imply that there is a one-to-one correspondence between $\mathrm{PSH}_{\omega}\left(\mathbb{P}^{n}\right)$ and $\mathcal{H}\left(\mathbb{C}^{n+1}\right)$.

Proposition 2.2.2. The map defined by

$$
\begin{aligned}
\operatorname{PSH}_{\omega}\left(\mathbb{P}^{n}\right) & \rightarrow \mathcal{L}\left(\mathbb{C}^{n}\right) \\
\varphi & \mapsto u(z):=\frac{1}{2} \log \left(1+|z|^{2}\right)+\varphi\left(1: z_{1}: \cdots: z_{n}\right), \quad z=\left(z_{1}, z_{2}, \cdots, z_{n}\right) \in \mathbb{C}^{n}
\end{aligned}
$$

is a bijection from $\operatorname{PSH}_{\omega}\left(\mathbb{P}^{n}\right)$ to $\mathcal{L}\left(\mathbb{C}^{n}\right)$.

Proof. Identify $\mathbb{C}^{n}$ with the affine open set $U_{0}$ :

$$
\mathbb{C}^{n} \simeq\left\{\left[1: \xi_{1}: \xi_{2}: \cdot: \xi_{n}\right] \in \mathbb{P}^{n}:\left(\xi_{1}, \ldots, \xi_{n}\right) \in \mathbb{C}^{n}\right\}=U_{0}
$$


Given $\varphi \in \operatorname{PSH}_{\omega}\left(\mathbb{P}^{n}\right)$. For $z=\left(z_{1}, z_{2}, \cdots, z_{n}\right) \in \mathbb{C}^{n}$, note that both $\log \left(1+|z|^{2}\right)$ and $\varphi\left(1: z_{1}: \cdots: z_{n}\right)$ belong to $\operatorname{PSH}\left(\mathbb{C}^{n}\right)$. Hence

$$
u(z):=\frac{1}{2} \log \left(1+|z|^{2}\right)+\varphi\left(1: z_{1}: \cdots: z_{n}\right) \in \operatorname{PSH}\left(\mathbb{C}^{n}\right)
$$

Since

$$
\sup \left(u(z)-(1 / 2) \log \left(1+|z|^{2}\right)\right)=\sup \left(\varphi\left(1: z_{1}: \cdots: z_{n}\right)\right)<\infty
$$

it follows that $u \in \mathcal{L}\left(\mathbb{C}^{n}\right)$.

Conversely, given $u \in \mathcal{L}\left(\mathbb{C}^{n}\right)$, for $\left(z_{0}: z\right) \in U_{0}$, define

$$
\begin{aligned}
\varphi_{0}\left(z_{0}: z\right)=\varphi_{0}\left(z_{0}: z_{1}: \cdots: z_{n}\right) & =\varphi_{0}\left(1: z_{1} / z_{0}: \cdots: z_{n} / z_{0}\right) \\
& =u\left(\frac{z}{z_{0}}\right)-\frac{1}{2} \log \left(1+\left|z / z_{0}\right|^{2}\right) \\
& =u\left(\frac{z}{z_{0}}\right)-\log \left|\left(1, \frac{z}{z_{0}}\right)\right|
\end{aligned}
$$

on a neighborhood $U$ of $U_{0}$. Then $\varphi_{0} \in \operatorname{PSH}(U)$ since both $u$ and $\log \left|\left(1, z / z_{0}\right)\right|$ are in $\operatorname{PSH}(U)$. Also,

$$
\begin{aligned}
d d^{c}\left(\varphi_{0}\right)+\omega & =d d^{c}\left(u\left(\frac{z}{z_{0}}\right)-\log \left|\left(1, z / z_{0}\right)\right|+\log \left|\left(z_{0}, z\right)\right|\right) \\
& =d d^{c}\left(u\left(\frac{z}{z_{0}}\right)+\log \left|z_{0}\right|\right) \geq 0
\end{aligned}
$$

shows that $\varphi_{0} \in \operatorname{PSH}_{\omega}(U)$. Since $u \in \mathcal{L}\left(\mathbb{C}^{n}\right), \sup \left(u\left(z / z_{0}\right)-\log \left|\left(1, z / z_{0}\right)\right|\right)<\infty$ implies that $\varphi_{0}$ is bounded above on $U$. Then, by Proposition 2.1.16, $\varphi_{0}$ can be extended to $\varphi \in$ $\operatorname{PSH}_{\omega}\left(\mathbb{P}^{n}\right)$. This completes the proof.

Proposition 2.2.3. There is a one-to-one correspondence between $\mathcal{H}\left(\mathbb{C}^{n+1}\right)$ and $\mathcal{L}\left(\mathbb{C}^{n}\right)$.

Proof. Given $h \in \mathcal{H}\left(\mathbb{C}^{n+1}\right)$. Let $u(z)=u\left(z_{1}, z_{2}, \ldots, z_{n}\right):=\log h\left(1, z_{1}, z_{2}, \ldots, z_{n}\right)$. By Theorem 2.2.1(ii),

$$
h=\left(\lim _{j \rightarrow \infty} \sup \left|Q_{j}\right|^{1 / j}\right)^{*}
$$


for some sequence $\left\{Q_{j} \in \mathscr{H}\left(\mathbb{C}^{n+1}\right)\right\}$ of complex homogeneous polynomials with $\operatorname{deg} Q_{j} \leq j$. Then

$$
\exp (u(z))=h(1, z)=\left(\lim _{j \rightarrow \infty} \sup \left|Q_{j}(1, z)\right|^{1 / j}\right)^{*}
$$

It follows from Theorem 2.2.1(iii) that $u(z) \in \mathcal{L}\left(\mathbb{C}^{n}\right)$.

Conversely, given $u \in \mathcal{L}\left(\mathbb{C}^{n}\right)$. Define

$$
h_{0}\left(z_{0}, z\right)=h_{0}\left(z_{0}, z_{1}, \ldots, z_{n}\right):=\left|z_{0}\right| \exp \left(u\left(z / z_{0}\right)\right)
$$

on $U_{0}$. Then $h_{0}\left(z_{0}, z\right) \in \operatorname{PSH}\left(U_{0}\right)$ since $u\left(z / z_{0}\right) \in \operatorname{PSH}\left(U_{0}\right)$. And

$$
h_{0}\left(\lambda z_{0}, \lambda z\right)=\left|\lambda z_{0}\right| \exp \left(u\left(\lambda z / \lambda z_{0}\right)\right)=|\lambda| h_{0}\left(z_{0}, z\right)
$$

implies that $h_{0}$ is non-negative homogeneous.

Since $u \in \mathcal{L}\left(\mathbb{C}^{n}\right)$, it follows that

$$
\begin{aligned}
\log h_{0}\left(z_{0}, z\right) & =u\left(z / z_{0}\right)+\log \left|z_{0}\right| \\
& \leq C+(1 / 2) \log \left(1+\left|z / z_{0}\right|^{2}\right)+\log \left|z_{0}\right| \\
& =C+\log \left|\left(z_{0}, z\right)\right|
\end{aligned}
$$

for some constant $C$. Hence, $h_{0} \in \operatorname{PSH}\left(U_{0}\right)$ is bounded above on $U_{0}$. Then by Proposition 2.1.16, we may extend $h_{0}$ to obtain $h \in \mathcal{H}\left(\mathbb{C}^{n+1}\right)$, as required.

\subsection{Pluripolar sets}

\subsubsection{Pluripolar sets in $\mathbb{C}^{n}$}

Definition 2.3.1. A Borel subset $F$ of $\mathbb{C}^{n}$ is said to be pluripolar (polar when $n=1$ ) if for each point $z \in F$ there is a plurisubharmonic function $u, u \neq \equiv-\infty$, defined in a connected neighborhood $U$ of $z$ in $\mathbb{C}^{n}$ such that $F \cap U \subset\{u=-\infty\}$. A set $F$ is said to be globally pluripolar if there is a non-constant plurisubharmonic function $u$ defined on $\mathbb{C}^{n}$ such that $F \subset\{y: u(y)=-\infty\}$. 
Note that a countable union of pluripolar sets is pluripolar. A classical result of Josefson (see [15]; see also [16, Theorem 4.7.4]) states that $F$ is pluripolar if and only if $F$ is globally pluripolar.

Definition 2.3.2. A subset $F \subset \mathbb{C}^{n}$ is said to be a complete pluripolar set if there is a non-constant plurisubharmonic function $u$ defined on $\mathbb{C}^{n}$ such that $F=\{u=-\infty\}$.

A subset $F$ of a domain $D$ in $\mathbb{C}^{n}$ is said to be a locally complete pluripolar set in $D$ if there is a non-constant plurisubharmonic function $u$ defined on $D$ such that $F=\{u=-\infty\}$. Note that each complete pluripolar set is $G_{\delta}$. When $n=1$, Deny's Theorem (see [6]) states that each $G_{\delta}$ polar set in $\mathbb{C}$ is a complete polar set.

Example 2.2. The set $F:=\left\{\left(0, z_{2}\right) \in \mathbb{C}^{2}:\left|z_{2}\right|<1\right\}$ and its closure are pluripolar, but they are not complete pluripolar sets. The set of rationals in the interval $[0,1]$ is polar. It is not a complete polar set, because each complete pluripolar set is $G_{\delta}$.

Example 2.3. The set $F:=\left\{\left(z_{1}, 0\right) \in \mathbb{C}^{2}:|z|<1\right\}$ is a complete pluripolar set in the bi-disk $\Delta_{2}:=\left\{\left(z_{1}, z_{2}\right) \in \mathbb{C}^{2}:\left|z_{1}\right|<1,\left|z_{2}\right|<1\right\}$, however, $F$ is not a complete pluripolar set in any domain $W$ with $\bar{\Delta}_{2} \subset W$.

Remark. From the above example we see that for a locally complete pluripolar set $F$ there does not necessarily exist a plurisubharmonic function $u$ defined in $\mathbb{C}^{n}$ such that $F=\{u=$ $-\infty$ \}. But it was shown in [5] that any closed locally pluripolar set in $\mathbb{C}^{n}$ is a complete pluripolar set.

Proposition 2.3.3. (see, e.g., [21]) If $F$ is a complete pluripolar set in $\mathbb{C}^{n}$ then there is a function $u \in \mathcal{L}\left(\mathbb{C}^{n}\right)$ such that $F=\{u=-\infty\}$.

Definition 2.3.4. The pluripolar hull (see [20]) of a pluripolar set $F$ in $\mathbb{C}^{n}$ is defined to be

$$
F^{*}=\cap\left\{z \in \mathbb{C}^{n}: u(z)=-\infty\right\},
$$

where the intersection is taken over all plurisubharmonic functions $u$ on $\mathbb{C}^{n}$ that are $-\infty$ on $F$. 
Remark. Some comments about pluripolar hulls in $\mathbb{C}^{n}$ may be in order.

(a) If $F$ is a complete pluripolar set, then $F^{*}=F$. The converse is not necessarily true. For example, $F=\left\{\left(z, z^{\nu}\right) \in \mathbb{C}^{2}: z \in \mathbb{C}, z \neq 0, \nu>0\right.$ is irrational $\}$, as a non- $G_{\delta}$ set, is not complete pluripolar, but $F^{*}=F$ (see [20]; see also [30] for details). However, the next Lemma, which is a result of Zeriahi (see [32, Proposition 2.1]), implies that if the pluripolar hull $F^{*}$ of a pluripolar set $F$ is both $F_{\sigma}$ and $G_{\delta}$ then $F^{*}$ is a complete pluripolar .

(b) The example in (a) implies that the pluripolar hull of a pluripolar set is in general not a complete pluripolar subset of $\mathbb{C}^{n}$.

Lemma 2.3.5. Let $F$ be a pluripolar set in $\mathbb{C}^{n}$. If $F$ is both $F_{\sigma}$ and $G_{\delta}$ and if $F^{*}=F$, then $F$ is a complete pluripolar set.

\subsubsection{Pluripolar sets in $\mathbb{P}^{n}$}

Let $\pi: \mathbb{C}^{n+1} \backslash\{0\} \rightarrow \mathbb{P}^{n}$ denote the standard projection mapping that maps a point $z=$ $\left(z_{0}, z_{1}, \ldots, z_{n}\right) \in \mathbb{C}^{n+1}$ to its corresponding homogeneous coordinates $\pi(z)=[z]=\left[z_{0}: z_{1}\right.$ : $\left.\ldots: z_{n}\right] \in \mathbb{P}^{n}$. Suppose that $z=\left(z_{0}, z_{1}, \ldots, z_{n}\right) \in \mathbb{C}^{n+1} \backslash\{0\}$ and $Z=\left[Z_{0}: Z_{1}: \cdots: Z_{n}\right] \in \mathbb{P}^{n}$. Then $Z=\pi(z)$ if and only if

$$
\left[Z_{0}: Z_{1}: \cdots: Z_{n}\right]=\left[z_{0}: z_{1}: \ldots: z_{n}\right]
$$

or, equivalently,

$$
z_{j} Z_{k}=z_{k} Z_{j}, \quad \text { for } j, k=0, \ldots, n
$$

Definition 2.3.6. A subset $E$ of $\mathbb{P}^{n}$ is said to be a pluripolar set in $\mathbb{P}^{n}$ if there is a function $\varphi \in \operatorname{PSH}_{\omega}\left(\mathbb{P}^{n}\right), \varphi \not \equiv-\infty$, such that $E \subset\{\varphi=-\infty\}$. A subset $K$ of $\mathbb{P}^{n}$ is said to be a complete pluripolar set in $\mathbb{P}^{n}$ if there is a non-constant function $\varphi \in \operatorname{PSH}_{\omega}\left(\mathbb{P}^{n}\right)$ such that $K=\{\varphi=-\infty\}$

Remark. The above definition does not depend on $\omega$; replacing $\omega$ by $c \omega$ with $c>0$ leads to an equivalent definition. In fact, a subset $E$ of $\mathbb{P}^{n}$ is a (complete) pluripolar set in $\mathbb{P}^{n}$ if and only if the set $U \cap E$ is a (complete) pluripolar set in $U$ for each affine open set $U$ (see, e.g., [21]). 


\subsection{Pluripolar hulls in $\mathbb{P}^{n}$}

Definition 2.4.1. The pluripolar hull (see, e.g., $[13,20]$ ) of a pluripolar set $\mathrm{E}$ in $\mathbb{P}^{n}$ is the intersection of all complete pluripolar sets in $\mathbb{P}^{n}$ that contain $E$, and is denoted by $E^{*}$, i.e.,

$$
E^{*}=\cap\left\{Z \in \mathbb{P}^{n}: \varphi(Z)=-\infty\right\},
$$

where the intersection is taken over all $\omega$-plurisubharmonic functions $\varphi$ on $\mathbb{P}^{n}$ that are $-\infty$ on $E$.

Remark. Notice first that $E \subset E^{*}$. For the case $n=1, E^{*}=E$. Indeed, since $E$ is polar in $\mathbb{P}^{n}$, there exists a $G_{\delta}$ polar set $G$ such that $E \subset G$. Let $Z \notin E$. By Deny's Theorem (see [6, p. 524]), $G \backslash\{Z\}$ is a complete polar set. It follows that there is a function $\varphi \in \mathrm{PSH}_{\omega}\left(\mathbb{P}^{1}\right)$ such that $G \backslash\{Z\}=\{\varphi=-\infty\}$. Hence, $\varphi(Z)>-\infty$, which implies $Z \notin E^{*}$. Thus $E^{*} \subset E$, as required.

In analogy with Lemma 2.3.5, we have the following Lemma.

Lemma 2.4.2. Let $E$ be a pluripolar set in $\mathbb{P}^{n}$. If $E$ is both $F_{\sigma}$ and $G_{\delta}$ and if $E^{*}=E$, then $E$ is a complete pluripolar set in $\mathbb{P}^{n}$.

Remark. The pluripolar hull of a pluripolar set is in general not a complete pluripolar set (see [20]). However, Lemma 2.4.2 implies that the pluripolar hull $E^{*}$ of a pluripolar set $E$ is a complete pluripolar set if $E^{*}$ is both $G_{\delta}$ and $F_{\sigma}$.

Proposition 2.4.3. If $E_{1} \subset E_{2} \subset \mathbb{P}^{n}$, then $E_{1}^{*} \subset E_{2}^{*}$.

Proof. This follows immediately from the definition.

Proposition 2.4.4. Let $\left\{E_{j}\right\}$ be a sequence of pluripolar sets in $\mathbb{P}^{n}$. Then

$$
\left(\cup_{j=1}^{\infty} E_{j}\right)^{*}=\cup_{j=1}^{\infty} E_{j}^{*}
$$

Proof. By Proposition 2.4.3, $\cup_{j=1}^{\infty} E_{j}^{*} \subset\left(\cup_{j=1}^{\infty} E_{j}\right)^{*}$. 
Suppose that $Z \notin \cup_{j=1}^{\infty} E_{j}^{*}$. Then $Z \notin E_{j}^{*}$ for each $j$, and hence there exists $\varphi_{j} \in$ $\operatorname{PSH}_{\omega}\left(\mathbb{P}^{n}\right)$ with $\left.\varphi_{j}\right|_{E_{j}}=-\infty, \varphi_{j}(Z)>-1$. Set $\varphi:=\sum_{j=1}^{\infty} 2^{-j} \varphi_{j}$. Then

$$
\varphi \in \operatorname{PSH}_{\omega}\left(\mathbb{P}^{n}\right), \varphi=-\infty \text { on } \cup_{j=1}^{\infty} E_{j}, \text { and }, \varphi(Z)>-1,
$$

which implies $Z \notin\left(\cup_{j=1}^{\infty} E_{j}\right)^{*}$.

Therefore, $\left(\cup_{j=1}^{\infty} E_{j}\right)^{*}=\cup_{j=1}^{\infty} E_{j}^{*}$. 


\section{CHAPTER 3}

\section{RELATIVE $\omega$-PLURISUBHARMONIC EXTREMAL FUNCTIONS}

We introduce the relative $\omega$-plurisubharmonic extremal functions on $\mathbb{P}^{n}$ which is analogous to the extremal functions in the local theory (see [4]).

\subsection{Relative $\omega$-Plurisubharmonic Extremal Functions}

Definition 3.1.1. For $E \subset \mathbb{P}^{n}, Z \in \mathbb{P}^{n}$, and $s \geq 0$, the relative $\omega$-plurisubharmonic extremal function with respect to the set $E$ and the parameter $s$ is defined by

$$
\sigma(Z, s, E)=\sup \left\{\varphi(Z): \varphi \in \operatorname{PSH}_{\omega}\left(\mathbb{P}^{n}\right), \varphi \leq-s \chi_{E}\right\}
$$

where $\chi_{E}$ is the characteristic function of $E$. Note that $\sigma(Z, 0, E)=0$.

Remark. The relative $\omega$-plurisubharmonic extremal function $\sigma(Z, s, E)$ defined above differs from the relative $\omega$-plurisubharmonic extremal function in [11] and the plurisubharmonic measure in $[3,26]$ in that it involves a parameter $s$. Note that $\sigma(Z, s, E)$ depends on $s$ in a nonlinear way, i.e., $\sigma(Z, s, E) \neq s \sigma(Z, 1, E)$. In [11], the authors considered $\sigma(Z, s, E)$ for the special case $s=1$. On the other hand, it is meaningless to involve the parameter $s$ in the corresponding definition in [26], since there the plurisubharmonic measure $\omega(z, E)$ was defined in terms of functions belonging to $\operatorname{PSH}\left(\mathbb{C}^{n}\right)$, which is a convex cone by Proposition 2.1.8 (i), and therefore would satisfy $\omega(z, s, E)=s \omega(z, 1, E)$.

For $F \subset \mathbb{C}^{n}, z \in \mathbb{C}^{n}$, and $s>0$, the relative extremal function with respect to the set $F$ and the parameter $s$ is defined by

$$
\sigma(z, s, F):=\sup \left\{u(z): u+(1 / 2) \log \left(1+|\cdot|^{2}\right) \in \operatorname{PSH}\left(\mathbb{C}^{n}\right), u \leq-s \chi_{F}\right\}
$$

Let $\iota: \mathbb{C}^{n} \rightarrow \mathbb{P}^{n}$ be defined by

$$
\iota\left(z_{1}, \cdots, z_{n}\right)=\left[1: z_{1}: \cdots: z_{n}\right]
$$


Then $\iota$ embeds $\mathbb{C}^{n}$ into $\mathbb{P}^{n}$ and identifies $\mathbb{C}^{n}$ with the affine open set

$$
\iota\left(\mathbb{C}^{n}\right)=U_{0}:=\left\{Z \in \mathbb{P}^{n}: Z_{0} \neq 0\right\} .
$$

Note that $\sigma(\iota(z), s, \iota(F))=\sigma(z, s, F)$.

For $E \subset \mathbb{P}^{n}$, let $\sigma^{*}(Z, s, E)$ denote the upper semicontinuous regularization of $\sigma(Z, s, E)$ with respect to $Z$. Then $\sigma^{*}(Z, s, E)$ is $\omega$-plurisubharmonic in $Z$, which is a straightforward consequence of the analogous local result for sequences of plurisubharmonic functions (see, e.g., $[16,11])$.

Proposition 3.1.2. Let $Z \in \mathbb{P}^{n}$. If $E_{1} \subset E_{2} \subset \mathbb{P}^{n}$, then $\sigma\left(Z, s, E_{2}\right) \leq \sigma\left(Z, s, E_{1}\right)$ and $\sigma^{*}\left(Z, s, E_{2}\right) \leq \sigma^{*}\left(Z, s, E_{1}\right)$ for each $Z \in \mathbb{P}^{n}$.

Proposition 3.1.2 is a direct consequence of the definition.

Proposition 3.1.3. If $E, P \subset \mathbb{P}^{n}$ and if $P$ is pluripolar, then $\sigma^{*}(Z, s, E)=\sigma^{*}(Z, s, E \cup P)$. In particular $\sigma^{*}(Z, s, P)=\sigma^{*}(Z, s, \varnothing)=0$ when $P$ is pluripolar.

Proof. It is enough to prove that $\sigma^{*}(Z, s, E) \leq \sigma^{*}(Z, s, E \cup P)$. Given $\delta>0$, let $\varepsilon>0$ be such that $(s+\delta) /(1+\varepsilon) \geq s$. Let $\varphi, \psi \in \operatorname{PSH}_{\omega}\left(\mathbb{P}^{n}\right)$ be such that $\varphi \leq-s \chi_{E}, \psi \leq 0, \psi \not \equiv-\infty$ and $\psi=-\infty$ on $P$. Then

$$
\frac{\varphi-\delta+\varepsilon \psi}{1+\varepsilon} \in \operatorname{PSH}_{\omega}\left(\mathbb{P}^{n}\right), \quad \frac{\varphi-\delta+\varepsilon \psi}{1+\varepsilon} \leq-s \chi_{E \cup P},
$$

which implies that

$$
\frac{\varphi-\delta+\varepsilon \psi}{1+\varepsilon} \leq \sigma^{*}(\cdot, s, E \cup P) .
$$

Letting $\varepsilon \rightarrow 0$, we obtain that $\varphi(Z)-\delta \leq \sigma^{*}(Z, s, E \cup P)$ for $Z \in \mathbb{P}^{n} \backslash W$ with $W:=\{\psi=$ $-\infty$. Note that $W$ has Lebesgue measure zero. It follows that, for each $Z \in \mathbb{P}^{n}$,

$$
\varphi(Z)-\delta \leq \sigma^{*}(Z, s, E \cup P),
$$

which implies that

$$
\sigma^{*}(Z, s, E)-\delta \leq \sigma^{*}(Z, s, E \cup P) .
$$

Letting $\delta \rightarrow 0$ yields that $\sigma^{*}(Z, s, E) \leq \sigma^{*}(Z, s, E \cup P)$, which completes the proof. 
Definition 3.1.4. (see, e.g., [16]) Let $F$ be a subset of $\mathbb{C}^{n}$ and let $\zeta \in \mathbb{C}^{n}$. Then $F$ is nonplurithin (or non-thin for the case $n=1$ ) at $\zeta$ if $\zeta \in \overline{F \backslash\{\zeta\}}$ and if, for each plurisubharmonic function $u$ defined on a neighborhood of $\zeta$,

$$
u(\zeta)=\limsup _{z \rightarrow \zeta, z \in F \backslash\{\zeta\}} u(z) .
$$

Otherwise we say that $F$ is plurithin (or thin for the case $n=1$ ) at $\zeta$.

Remark. Let $u$ be a plurisubharmonic function on a neighborhood of $\zeta \in \mathbb{C}^{n}$. We certainly have $\lim \sup _{z \rightarrow \zeta} u(z)=u(\zeta)$. It follows that a set $F$ is non-plurithin at each point of its interior. In particular, an open set is non-plurithin at each point of itself.

For $E \subset \mathbb{P}^{n}$, let

$$
G:=\left\{Y \in E: \sigma^{*}(Y, s, E)>-s\right\} .
$$

Then by Proposition 2.1.10, the negligible set $G$ is pluripolar. Since $\sigma^{*}(Z, s, E) \leq-s \chi_{E \backslash G}$, we have

$$
\sigma^{*}(Z, s, E) \leq \sigma(Z, s, E \backslash G) \leq \sigma^{*}(Z, s, E \backslash G) .
$$

Thus by Proposition 3.1.3,

$$
\sigma^{*}(Z, s, E)=\sigma(Z, s, E \backslash G) .
$$

Denote by $E^{\circ}$ the interior of $E$.

Proposition 3.1.5. Let $E \subset \mathbb{P}^{n}$ be such that $E^{\circ}$ is non-plurithin at each point of $E$. Then $\sigma^{*}(Z, s, E)=\sigma(Z, s, E)$ for each $Z \in \mathbb{P}^{n}$.

Proof. It suffices to show that $\sigma^{*}(Z, s, E) \leq \sigma(Z, s, E)$ for each $Z \in \mathbb{P}^{n}$. Since $\sigma(\cdot, s, E)=$ $-s$ on $E$, we have $\sigma^{*}(\cdot, s, E)=-s$ on $E^{\circ}$. By definition if $E^{\circ}$ is non-plurithin at $Z$, then $\sigma^{*}(Z, s, E)=-s$. Hence, the assumption that $E^{\circ}$ is non-plurithin at each point of $E$ implies that $\sigma^{*}(\cdot, s, E)=-s$ on E. It follows that $\sigma^{*}(Z, s, E) \leq \sigma(Z, s, E)$, which completes the proof. 
Corollary 3.1.6. If $E \subset \mathbb{P}^{n}$ is open, then $\sigma^{*}(Z, s, E)=\sigma(Z, s, E)$ for each $Z \in \mathbb{P}^{n}$.

As an example we now give explicit formulae for $\sigma^{*}(z, s, \rho \bar{B})$, where $z \in \mathbb{C}^{n}, B=\{z \in$ $\left.\mathbb{C}^{n}:|z|<1\right\}$, and $\rho \bar{B}=\left\{z \in \mathbb{C}^{n}:|z| \leq \rho\right\}$. It is an immediate consequence of the definition that $\sigma^{*}(z, 0, F)=0$ for each subset $F$ of $\mathbb{C}^{n}$. By Proposition 3.1.5, $\sigma^{*}(z, s, F)=-s$ for each point $z$ at which the interior $F^{\circ}$ is non-plurithin. Thus $\sigma^{*}(z, s, \rho \bar{B})=-s$ for $|z| \leq \rho$. So it suffices to calculate $\sigma^{*}(z, s, \rho \bar{B})$ for $s>0$ and $|z|>\rho$.

Example 3.1. Let $s>0$ be a fixed positive number and consider

$$
u(z):=\sigma^{*}(z, s, \rho \bar{B})+(1 / 2) \log \left(1+|z|^{2}\right), z \geq \rho
$$

Then the function $u(z)$ is given by

$$
u(z)= \begin{cases}(1 / 2) \log \left(1+\rho^{2}\right)-s+\log (|z| / \rho), & \text { if } s \geq(1 / 2) \log \left(1+\rho^{-2}\right), \\ (1 / 2) \log \left(1+\rho^{2}\right)-s+\frac{\log (|z| / \rho)}{1+e^{-2 \lambda},} & \text { if } s<(1 / 2) \log \left(1+\rho^{-2}\right), \quad|z| \leq e^{\lambda}, \\ (1 / 2) \log \left(1+|z|^{2}\right), & \text { if } s<(1 / 2) \log \left(1+\rho^{-2}\right), \quad|z|>e^{\lambda},\end{cases}
$$

where $\lambda=\lambda(\rho, s)$ is the unique number in the interval $(\log \rho, \infty)$ that satisfies the equation

$$
\frac{e^{2 \lambda}}{1+e^{2 \lambda}}=\left(\frac{1}{2} \log \frac{1+e^{2 \lambda}}{1+\rho^{2}}+s\right) /(\lambda-\log \rho) .
$$

Proof. Notice first that $u(z) \in \operatorname{PSH}\left(\mathbb{C}^{n}\right)$ and for all $z \in \mathbb{C}^{n}$,

$$
u(z) \leq \frac{1}{2} \log \left(1+|z|^{2}\right) .
$$

Since $u(z)=-s+(1 / 2) \log \left(1+\rho^{2}\right)$ on $\{|z|=\rho\}$, and since the Siciak's extremal function of $\rho \bar{B}$ is $\log ^{+}(|z| / \rho)$ (see [16, p. 185]), it follows that

$$
u(z) \leq-s+\frac{1}{2} \log \left(1+\rho^{-2}\right)+\log |z| \quad \text { for }|z| \geq \rho .
$$

Moreover, since the set $\rho \bar{B}$ is "radial", the function $u$ is radial. Set $v(t)=u\left(e^{t}, 0, \ldots, 0\right)$ for $t \geq \log \rho$. Then $u(z)=v(\log |z|)$. The function $v(t)$ satisfies

$$
\begin{aligned}
& v(t) \leq \frac{1}{2} \log \left(1+e^{2 t}\right) \\
& v(t) \leq-s+\frac{1}{2} \log \left(1+\rho^{-2}\right)+t .
\end{aligned}
$$


Since $u(z)$ is plurisubharmonic, the function $v(t)$ is increasing and convex (see [23, p. 45]). Therefore, $v$ is the greatest convex function that does not exceed the function $w(t)$, where

$$
\begin{gathered}
w(t):=\min \left\{\mu_{1}(t), \mu_{2}(t)\right\}, \\
\mu_{1}(t):=\frac{1}{2} \log \left(1+e^{2 t}\right), \quad \mu_{2}(t):=-s+\frac{1}{2} \log \left(1+\rho^{-2}\right)+t .
\end{gathered}
$$

Both $\mu_{1}$ and $\mu_{2}$ are increasing and convex, so $w(t)$ is increasing; but $w(t)$ is not necessarily convex.

When $s \geq(1 / 2) \log \left(1+\rho^{-2}\right)$, we have $\mu_{2}(t) \leq t<\mu_{1}(t)$, so $w(t)=\mu_{2}(t)$ is convex, and therefore $v(t)=w(t)=\mu_{2}(t)$.

We now consider the case $s<(1 / 2) \log \left(1+\rho^{-2}\right)$. The function $\mu(t):=\mu_{2}(t)-\mu_{1}(t)$ satisfies $\mu(\log \rho)=-s<0, \lim _{t \rightarrow \infty} \mu(t)=-s+(1 / 2) \log \left(1+\rho^{-2}\right)>0$, and $\mu^{\prime}(t)>0$. It follows that there is a unique point $t_{0} \in(\log \rho, \infty)$ such that $\mu\left(t_{0}\right)=0$. Thus

$$
w(t)= \begin{cases}\mu_{2}(t), & \text { if } \log \rho \leq t \leq t_{0}, \\ \mu_{1}(t), & \text { if } t_{0} \leq t .\end{cases}
$$

Since $w^{\prime}(t)=1$ on $\left(\log \rho, t_{0}\right)$ and $w^{\prime}(t)<1$ on $\left(t_{0}, \infty\right)$, it follows that $w$ is not convex on $(\log \rho, \infty)$. Consider the function

$$
\tau(t):=\frac{\mu_{1}(t)-\mu_{2}(\log \rho)}{t-\log \rho}, \quad t \geq t_{0},
$$

which is the slope of the line segment from $\left(\log \rho, \mu_{2}(\log \rho)\right)$ to $\left(t, \mu_{1}(t)\right)$. Since $\tau\left(t_{0}\right)=1=$ $\lim _{t \rightarrow \infty} \tau(t)$, and since $\tau(t)<1$ for $t>t_{0}$, we see that $\tau(t)$ must attain its minimum at some $\lambda>t_{0}$. Now $\tau(\lambda)$ is necessarily equal to $\mu_{1}^{\prime}(\lambda)$, and hence the strict convexity of $\mu_{1}$ implies the uniqueness of $\lambda$. Since $\tau(\lambda)=\mu_{1}^{\prime}(\lambda)$, the unique number $\lambda$ is determined by (3.4). Consequently, the function $v(t)$ is given by

$$
v(t)= \begin{cases}\mu_{2}(\log \rho)+\tau(\lambda)(t-\log \rho), & \text { if } \log \rho \leq t \leq \lambda, \\ \mu_{1}(t), & \text { if } \lambda \leq t .\end{cases}
$$

In summary, combining the two cases, we obtain

$$
v(t)= \begin{cases}(1 / 2) \log \left(1+\rho^{2}\right)-s+t-\log \rho, & \text { if } s \geq(1 / 2) \log \left(1+\rho^{-2}\right), \\ (1 / 2) \log \left(1+\rho^{2}\right)-s+\left(1+e^{-2 \lambda}\right)^{-1}(t-\log \rho), & \text { if } s<(1 / 2) \log \left(1+\rho^{-2}\right), t \leq \lambda, \\ (1 / 2) \log \left(1+e^{2 t}\right), & \text { if } s<(1 / 2) \log \left(1+\rho^{-2}\right), t>\lambda,\end{cases}
$$


where $\lambda$ satisfies (3.4). The proof of (3.3) is complete. This gives explicit formulae for $\sigma^{*}(z, s, \rho \bar{B})$ because it is equal to $u(z)-(1 / 2) \log \left(1+|z|^{2}\right)$.

Remark. By the above implicit expression of the unique number $\lambda(\rho, s)$ which depends on $\rho$ and $s$, we obtain that

$$
\frac{\partial \lambda}{\partial \rho}=\frac{1+e^{2 \lambda}}{2 \rho e^{2 \lambda}\left(1+\rho^{2}\right)} \cdot \frac{e^{2 \lambda}-\rho^{2}}{\lambda-\log \rho}>0 .
$$

Hence $\lambda(\rho, s)$ is increasing with respect to $\rho$, i.e., $\lambda\left(\rho_{1}, s\right)<\lambda\left(\rho_{2}, s\right)$ if $\rho_{1}<\rho_{2}$.

Proposition 3.1.7. If $\left\{E_{j}\right\}$ is an ascending sequence of subsets in $\mathbb{P}^{n}$ and $E=\cup_{j=1}^{\infty} E_{j}$, then $\sigma^{*}\left(Z, s, E_{j}\right)$ decreases towards $\sigma^{*}(Z, s, E)$ for each $Z \in \mathbb{P}^{n}$.

Proof. By Proposition 3.1.3, $\sigma^{*}\left(Z, s, E_{j}\right)=0$ if $E_{j}$ is pluripolar. Thus we may assume that $E_{1}$ is not pluripolar. The decreasing sequence $\left\{\sigma^{*}\left(Z, s, E_{j}\right)\right\}$ of $\omega$-plurisubharmonic functions has an $\omega$-plurisubharmonic $\operatorname{limit}_{j \rightarrow \infty} \sigma^{*}\left(Z, s, E_{j}\right)$. Set $\varphi:=\lim _{j \rightarrow \infty} \sigma^{*}\left(Z, s, E_{j}\right)$. Then $\varphi \in \operatorname{PSH}_{\omega}\left(\mathbb{P}^{n}\right)$ and $\sigma^{*}(Z, s, E) \leq \varphi(Z)$. We now prove the reversed inequality $\varphi(Z) \leq$ $\sigma^{*}(Z, s, E)$. Put

$$
G:=\cup_{j=1}^{\infty}\left\{Y \in E_{j}: \sigma^{*}\left(Y, s, E_{j}\right)>-s\right\}
$$

Then $\mathrm{G}$ is pluripolar. Note that $\varphi=-s$ on $E \backslash G$, which implies that

$$
\varphi(Z) \leq \sigma(Z, s, E \backslash G)
$$

By (3.2),

$$
\varphi(Z) \leq \sigma^{*}(Z, s, E)
$$

Therefore, $\lim _{j \rightarrow \infty} \sigma^{*}\left(Z, s, E_{j}\right)=\sigma^{*}(Z, s, E)$. This completes the proof.

Proposition 3.1.8. If $\left\{K_{j}\right\}$ is a descending sequence of compact subsets in $\mathbb{P}^{n}$ and $K=$ $\cap_{j=1}^{\infty} K_{j}$, then $\sigma\left(Z, s, K_{j}\right)$ increases towards $\sigma(Z, s, K)$ for each $Z \in \mathbb{P}^{n}$. 
Proof. Noting that $\lim _{j \rightarrow \infty} \sigma\left(Z, s, K_{j}\right)$ exists and that $\lim _{j \rightarrow \infty} \sigma\left(Z, s, K_{j}\right) \leq \sigma(Z, s, K)$, it suffices to show that $\sigma(Z, s, K) \leq \lim _{j \rightarrow \infty} \sigma\left(Z, s, K_{j}\right)$. Let $\varphi \in \operatorname{PSH}_{\omega}\left(\mathbb{P}^{n}\right)$ be such that $\varphi \leq-s \chi_{K}$. Given $\varepsilon>0$. By the compactness of $K_{j}, K_{j}$ is contained in the open set $\left\{Z \in \mathbb{P}^{n}: \varphi(Z)<-s+\varepsilon\right\}$ for sufficiently large $j$. Thus, for such values of $j$,

$$
\varphi(Z)-\varepsilon \leq \sigma\left(Z, s, K_{j}\right) \leq \lim _{j \rightarrow \infty} \sigma\left(Z, s, K_{j}\right), \quad Z \in \mathbb{P}^{n} .
$$

Consequently, $\sigma(Z, s, K) \leq \lim _{j \rightarrow \infty} \sigma\left(Z, s, K_{j}\right)+\varepsilon$. Letting $\varepsilon \rightarrow 0$ yields $\sigma(Z, s, K) \leq$ $\lim _{j \rightarrow \infty} \sigma\left(Z, s, K_{j}\right)$, as desired.

Remark. (a) For Proposition 3.1.7, we do not know whether $\sigma\left(Z, s, E_{j}\right)$ decreases towards $\sigma(Z, s, E)$ when $n \geq 2$, but we have the following Proposition 3.1.9 for the case $n=1$.

(b) For Proposition 3.1.8, the following Example 3.2 implies that $\lim _{j \rightarrow \infty} \sigma^{*}\left(Z, s, K_{j}\right) \neq$ $\sigma^{*}(Z, s, K)$ in general.

Proposition 3.1.9. Let $\left\{E_{j}\right\}$ be an ascending sequence of subsets of $\mathbb{P}^{1}$, and let $E:=$ $\cup_{j=1}^{\infty} E_{j}$. Then

$$
\lim _{j \rightarrow \infty} \sigma\left(Z, s, E_{j}\right)=\sigma(Z, s, E), \quad Z \in \mathbb{P}^{n} .
$$

Proof. If $Z \in E$, then there exists some $j_{0}$ such that $Z \in E_{j}$ for each $j>j_{0}$, and hence $\lim _{j \rightarrow \infty} \sigma\left(Z, s, E_{j}\right)=\sigma(Z, s, E)=-s$.

Now suppose that $Z \notin E$. It is enough to show that $\lim _{j \rightarrow \infty} \sigma\left(Z, s, E_{j}\right) \leq \sigma(Z, s, E)$. Put

$$
G:=\left\{Y \in E: \sigma^{*}(Y, s, E)>-s\right\} .
$$

Then $G$ is polar. We claim that

$$
\sigma(Z, s, E)=\sigma(Z, s, E \backslash G) .
$$

In fact, by Proposition 3.1.2, we have $\sigma(Z, s, E) \leq \sigma(Z, s, E \backslash G)$. Take $\varepsilon>0$. Let $\varphi \in$ $\operatorname{PSH}_{\omega}\left(\mathbb{P}^{1}\right)$ be such that $\varphi \leq-s \chi_{E \backslash G}, \varphi(Z)>\sigma(Z, s, E \backslash G)-\varepsilon$. Since $G$ is polar, Deny's 
Theorem (see [6, p. 524]) provides a function $\psi \in \mathrm{PSH}_{\omega}\left(\mathbb{P}^{1}\right)$ such that $\psi \leq-s$ on $\mathbb{P}^{1}, \psi=$ $-\infty$ on $G$ and $\psi(Z)>-\infty$. Set $a:=\sigma(Z, s, E \backslash G), b:=\psi(Z)$, and put $\varphi_{\varepsilon}=(1-\varepsilon) \varphi+\varepsilon \psi$. Then $\varphi_{\varepsilon} \in \mathrm{PSH}_{\omega}\left(\mathbb{P}^{1}\right), \varphi_{\varepsilon} \leq 0$ on $\mathbb{P}^{1}$, and $\varphi_{\varepsilon} \leq-s$ on $E$. Hence, $\sigma(Z, s, E) \geq \varphi_{\varepsilon}(Z)>$ $(1-\varepsilon)(a-\varepsilon)+\varepsilon b$. Letting $\varepsilon \rightarrow 0$ yields $\sigma(Z, s, E) \geq a$, which shows $\sigma(Z, s, E)=\sigma(Z, s, E \backslash G)$. Therefore, in view of (3.2),

$$
\lim _{j \rightarrow \infty} \sigma\left(Z, s, E_{j}\right) \leq \lim _{j \rightarrow \infty} \sigma^{*}\left(Z, s, E_{j}\right)=\sigma^{*}(Z, s, E)=\sigma(Z, s, E \backslash G)=\sigma(Z, s, E),
$$

as required.

Example 3.2. Let $K_{j}:=(1 / j) \bar{B}$. Then by Proposition 3.1 .5 for each $j, \sigma^{*}\left(z, s, K_{j}\right)=$ $\sigma\left(z, s, K_{j}\right)$ since $K_{j}^{\circ}$ is non-plurithin at each point of $K_{j}$. Fixing $s$, by Example 3.1 and the following remark, we have $\lambda(j, s)<\lambda(\ell, s)$ when $j>\ell$. Hence we may claim that $\lim _{j \rightarrow \infty} \lambda(j, s)=-\infty$. In fact, if $\lim _{j \rightarrow \infty} \lambda(j, s)=\lambda_{0} \neq-\infty$, then

$$
0<\frac{e^{2 \lambda_{0}}}{1+e^{2 \lambda_{0}}}=\lim _{j \rightarrow \infty} \frac{\frac{1}{2} \log \left(1+e^{2 \lambda_{0}}\right)+s}{\lambda_{0}-\log (1 / j)}=0,
$$

a contradiction. It follows that

$$
\lim _{j \rightarrow \infty} \sigma^{*}(z, s,(1 / j) \bar{B})= \begin{cases}0, & \text { for } z \neq 0 \\ -s, & \text { for } z=0 .\end{cases}
$$

On the other hand, we have that $\sigma(0, s,\{0\})=-s$ and $\sigma(z, s,\{0\}) \leq 0$. For $z \neq 0$, let $\varphi(z):=\log |z|-(1 / 2) \log \left(1+|z|^{2}\right)$. Then $\varphi \in \operatorname{PSH}_{\omega}\left(\mathbb{P}^{n}\right), \varphi \leq 0$ on $\mathbb{P}^{n}$ and $\varphi(0)=-\infty$.

Given $0<\delta<1$, we have $\delta \varphi \in \operatorname{PSH}_{\omega}\left(\mathbb{P}^{n}\right)$,

$$
\delta \varphi \leq 0 \text { on } \mathbb{P}^{n}, \delta \varphi(0) \leq-s,
$$

which implies that $\delta \varphi(z) \leq \sigma(z, s,\{0\})$. Letting $\delta \rightarrow 0$ yields $0 \leq \sigma(z, s,\{0\})$ for $z \neq 0$. Hence,

$$
\sigma(z, s,\{0\})= \begin{cases}0, & \text { for } z \neq 0 \\ -s, & \text { for } z=0 .\end{cases}
$$

This implies $\sigma^{*}(z, s,\{0\})=0$.

Therefore,

$$
\sigma^{*}(z, s,\{0\}) \neq \lim _{j \rightarrow \infty} \sigma^{*}(z, s,(1 / j) \bar{B}) .
$$


As a function of $s, \sigma(Z, s, E)$ has the following property.

Proposition 3.1.10. For fixed $Z, E, \sigma(Z, s, E)$ is a decreasing, concave function of $s$.

Proof. Fixing $Z$ and $E$, it follows straightforwardly from the definition that $\sigma(Z, s, E)$ is decreasing in $s$.

Let $s_{1}, s_{2} \geq 0$ and $\lambda \in[0,1]$. Given $\varepsilon>0$, there exist $\varphi_{1}, \varphi_{2} \in \operatorname{PSH}_{\omega}\left(\mathbb{P}^{n}\right)$ with $\varphi_{1} \leq$ $-s_{1} \chi_{E}$ and $\varphi_{2} \leq-s_{2} \chi_{E}$ such that

$$
\varphi_{1}(Z)>\sigma\left(Z, s_{1}, E\right)-\varepsilon \text { and } \varphi_{2}(Z)>\sigma\left(Z, s_{2}, E\right)-\varepsilon
$$

Set $\varphi:=(1-\lambda) \varphi_{1}+\lambda \varphi_{2}$. Then $\varphi \in \operatorname{PSH}_{\omega}\left(\mathbb{P}^{n}\right)$. Since

$$
\begin{aligned}
\varphi & \leq-\left((1-\lambda) s_{1}+\lambda s_{2}\right) \chi_{E}, \\
\varphi(Z) & >(1-\lambda)\left(\sigma\left(Z, s_{1}, E\right)-\varepsilon\right)+\lambda\left(\sigma\left(Z, s_{2}, E\right)-\varepsilon\right),
\end{aligned}
$$

it follows that

$$
\sigma\left(Z,(1-\lambda) s_{1}+\lambda s_{2}, E\right) \geq \varphi(Z)>(1-\lambda) \sigma\left(Z, s_{1}, E\right)+\lambda \sigma\left(Z, s_{2}, E\right)-\varepsilon
$$

Letting $\varepsilon \rightarrow 0$ yields that

$$
\sigma\left(Z,(1-\lambda) s_{1}+\lambda s_{2}, E\right) \geq(1-\lambda) \sigma\left(Z, s_{1}, E\right)+\lambda \sigma\left(Z, s_{2}, E\right)
$$

Therefore, $\sigma(Z, s, E)$ is concave in $s$.

Proposition 3.1.11. Let $E$ be a subset of $\mathbb{P}^{n}$ and let $Z \in \mathbb{P}^{n} \backslash E$. Then $\sigma(Z, s, E)=0$ for each $s>0$ if and only if there is a positive number $\tau$ such that $\sigma(Z, s, E) \geq-\tau$ for each $s>0$.

Proof. It suffices to show the sufficiency. Let $\tau>0$ be such that $\sigma(Z, s, E) \geq-\tau$ for each $s>0$. Let $\varepsilon>0$ be given. Set $\alpha=(\tau+\varepsilon) / \varepsilon$. Since $\sigma(Z, \alpha s, E) \geq-\tau$, we see that there is a function $\varphi \in \operatorname{PSH}_{\omega}\left(\mathbb{P}^{n}\right)$ such that

$$
\varphi \leq-\alpha s \chi_{E} \text { and } \varphi(Z)>-\tau-\varepsilon
$$


Now $\alpha^{-1} \varphi \in \operatorname{PSH}_{\omega}\left(\mathbb{P}^{n}\right), \alpha^{-1} \varphi \leq-s \chi_{E}$, and

$$
\alpha^{-1} \varphi(Z)>\alpha^{-1}(-\tau-\varepsilon)=-\varepsilon
$$

It follows that $\sigma(Z, s, E)>-\varepsilon$. Letting $\varepsilon \rightarrow 0$ yields that $\sigma(Z, s, E) \geq 0$, which completes the proof.

\subsection{Pluripolar hulls in terms of $\omega$-plurisubharmonic functions}

Set

$$
E^{(0)}:=\left\{Z \in \mathbb{P}^{n}: \sigma(Z, s, E)=0 \text { for each } s>0\right\}
$$

Theorem 3.2.1. Let $E$ be a pluripolar set in $\mathbb{P}^{n}$. Then $E^{(0)}$ is the complement of $E^{*}$.

Proof. Suppose that $Z \notin E^{*}$. Then there exists a function $\varphi \in \operatorname{PSH}_{\omega}\left(\mathbb{P}^{n}\right)$ such that

$$
\varphi \leq 0,\left.\quad \varphi\right|_{E}=-\infty, \text { and } \varphi(Z)>-\infty
$$

Let $s, \varepsilon>0$ be given. Choose $\delta \in(0,1)$ so that $\delta \varphi(Z)>-\varepsilon$. Note that $\delta \varphi \in \mathrm{PSH}_{\omega}\left(\mathbb{P}^{n}\right)$ and $\delta \varphi \leq-s \chi_{E}$, which implies that $\sigma(Z, s, E)>-\varepsilon$. Letting $\varepsilon \rightarrow 0$ yields that $\sigma(Z, s, E)=0$. This means that $Z \in E^{(0)}$.

Conversely, assume that $Z \in E^{(0)}$. Then $\sigma\left(Z, 2^{j}, E\right)=0$ for each $j \in \mathbb{N}$. It follows that there is a sequence of functions $\left\{\varphi_{j}\right\} \subset \operatorname{PSH}_{\omega}\left(\mathbb{P}^{n}\right)$ with $\varphi_{j} \leq-2^{j} \chi_{E}$ and $\varphi_{j}(Z)>-1$. Setting $\varphi:=\sum_{j=1}^{\infty} 2^{-j} \varphi_{j} \in \operatorname{PSH}_{\omega}\left(\mathbb{P}^{n}\right)$, we obtain

$$
\begin{gathered}
\left.\varphi\right|_{E} \leq \sum_{j=1}^{\infty}(-1)=-\infty, \\
\varphi(Z)>-\sum_{j=1}^{\infty} 2^{-j}=-1 .
\end{gathered}
$$

Thus $Z \notin E^{*}$, which completes the proof.

The following Corollary can be derived immediately from Proposition 2.4.4 and Theorem 3.2.1. 
Corollary 3.2.2. Let $\left\{E_{j}\right\}_{j=1}^{k}$ be a sequence of pluripolar sets in $\mathbb{P}^{n}$. Then

$$
\left(\cup_{j=1}^{k} E_{j}\right)^{(0)}=\cap_{j=1}^{k} E_{j}^{(0)}
$$

Let $E$ be a pluripolar set in $\mathbb{P}^{n}$. For $\mu, \beta>0$, put

$$
\begin{gathered}
E_{\mu, \beta}:=\left\{Z \in \mathbb{P}^{n}: \sigma(Z, s, E) \leq \beta-\frac{1}{\mu} s, \text { for each } s>0\right\} \\
E_{\mu, \infty}:=\cup_{\beta>0} E_{\mu, \beta} .
\end{gathered}
$$

Theorem 3.2.3. $E^{*}=\cup_{\mu>0} E_{\mu, \infty}$.

Proof. Suppose that $Z \in E^{*}$. By Theorem 3.2.1 there is an $s_{0}>0$ such that $\beta:=$ $-\sigma\left(Z, s_{0}, E\right)>0$. Let $\mu=s_{0} / \beta$. We now show that $\sigma(Z, s, E) \leq \beta-s / \mu$ for each $s>0$, which implies that $Z \in E_{\mu, \beta}$. If $s \leq s_{0}$, then $\sigma(Z, s, E) \leq 0=\beta-s_{0} / \mu \leq \beta-s / \mu$. Now we assume that $s>s_{0}$. Set $\lambda=s_{0} / s$. By the concavity of $\sigma(Z, s, E)$ we have

$$
\begin{aligned}
\sigma(Z, s, E) & =(1 / \lambda)(\lambda \sigma(Z, s, E)+(1-\lambda) \sigma(Z, 0, E)) \\
& \leq(1 / \lambda) \sigma(Z, \lambda s, E) \\
& =-\beta / \lambda=-s / \mu .
\end{aligned}
$$

Thus $\sigma(Z, s, E)<\beta-s / \mu$, which means $Z \in E_{\mu, \beta}$. Hence $E^{*} \subset \cup_{\mu>0} E_{\mu, \infty}$

Conversely, assume that $Z \in E_{\mu, \beta}$ for some $\mu, \beta$. Then $\sigma(Z, s, E) \leq \beta-s / \mu$, which implies that $\sigma(Z, s, E)<0$ for large enough $s$. Hence, $Z \in E^{*}$ by Theorem 3.2.1. Therefore, $\cup_{\mu>0} E_{\mu, \infty} \subset E^{*}$. This completes the proof.

Remark. For $m \in \mathbb{N}, \cup_{m=1}^{\infty} E_{m, \infty}=\cup_{m=1}^{\infty} E_{m, m}$. Setting $E^{(m)}:=E_{m, m}$, we have

$$
E \subset E^{(m)}, \quad E^{(m)} \subset E^{(m+1)}, \quad \text { and } \quad E^{*}=\cup_{m=1}^{\infty} E^{(m)} .
$$




\section{CHAPTER 4 \\ PROPERTY J in $\mathbb{P}^{n}$}

Property J defined in [21] for a set in $\mathbb{C}^{n}$ is the very property one uses to prove that the set is a convergence set. We now introduce Property $\mathrm{J}$ in $\mathbb{P}^{n}$, which will be used to establish a connection between the union of the pluripolar hulls of a countable collections of compact pluripolar sets and a convergence set in $\mathbb{P}^{n}$ (see Theorem 5.1.5).

\subsection{Property $\mathrm{J}$ in $\mathbb{P}^{n}$}

For $p \in \mathscr{H}_{k}\left(\mathbb{C}^{n+1}\right)$ with $k \geq 1, z \in \mathbb{C}^{n+1} \backslash\{0\}$ and $Z=[z] \in \mathbb{P}^{n}$, set

$$
\langle p(Z)\rangle:=\frac{|p(Z)|^{1 / k}}{|Z|}=\frac{|p(z)|^{1 / k}}{|z|} .
$$

For $p \in \mathscr{H}_{0}\left(\mathbb{C}^{n+1}\right)$, let $\langle p(Z)\rangle=|p(Z)|$. Note that $\langle p(Z)\rangle$ is independent of the choice of the representative $z$ and is a well-defined function on $\mathbb{P}^{n}$. Furthermore, if $m, k>0$ and $p \in \mathscr{H}_{k}\left(\mathbb{C}^{n}\right)$, then $\left\langle p^{m}(Z)\right\rangle=\langle p(Z)\rangle$. For a set $E \subset \mathbb{P}^{n}$, put

$$
\langle p\rangle_{E}=\sup _{Z \in E}\langle p(Z)\rangle, \quad\langle p\rangle=\langle p\rangle_{\mathbb{P}^{n}} .
$$

Definition 4.1.1. A subset $E$ of $\mathbb{P}^{n}$ is said to have Property $J$ (in $\mathbb{P}^{n}$ ) with respect to a point $X \in \mathbb{P}^{n} \backslash E$ if there is a sequence $\left\{p_{j}\right\} \subset \mathscr{H}\left(\mathbb{C}^{n+1}\right)$ and $0<\eta<1$ such that

$$
\left\langle p_{j}(X)\right\rangle>\eta, \quad\left\langle p_{j}\right\rangle \leq 1, \quad \lim _{j \rightarrow \infty}\left\langle p_{j}\right\rangle_{E}=0 .
$$

The set $E$ is said to have Property $J$ if $E$ has Property $J$ with respect to each $X \in \mathbb{P}^{n} \backslash E$.

Proposition 2.2.2 and 2.2.3 imply that there is a one to one correspondence between $\operatorname{PSH}_{\omega}\left(\mathbb{P}^{n}\right)$ and $\mathcal{H}\left(\mathbb{C}^{n+1}\right)$. Given $h(z) \in \mathcal{H}\left(\mathbb{C}^{n+1}\right)$ and $Z=\pi(z)$, the function

$$
\varphi(Z):=\log h(z)-\log |z|
$$

belongs to $\operatorname{PSH}_{\omega}\left(\mathbb{P}^{n}\right)$, and $h \mapsto \varphi$ is a bijection from $\mathcal{H}\left(\mathbb{C}^{n+1}\right)$ to $\operatorname{PSH}_{\omega}\left(\mathbb{P}^{n}\right)$.

By Theorem 2.2.1 (i) or [31, Proposition 2.10] we have the following Lemma. 
Lemma 4.1.2. If $\varphi \in C\left(\mathbb{P}^{n}\right) \cap \operatorname{PSH}_{\omega}\left(\mathbb{P}^{n}\right)$ and $\varphi>-\infty$, then

$$
\varphi(Z)=\sup \left\{\log \langle p(Z)\rangle: p \in \mathscr{H}\left(\mathbb{C}^{n+1}\right), \log \langle p\rangle \leq \varphi \text { on } \mathbb{P}^{n}\right\}, \quad Z \in \mathbb{P}^{n}
$$

Proposition 4.1.3. Let $K$ be a compact set in $\mathbb{P}^{n}$ and $Z \in \mathbb{P}^{n}$. For $0<t \leq 1$, define

$$
Q_{K, Z}(t)=\sup \left\{\langle p(Z)\rangle: p \in \mathscr{H}\left(\mathbb{C}^{n+1}\right),\langle p\rangle \leq 1,\langle p\rangle_{K} \leq t\right\}
$$

Then $\sigma(Z, s, K)=\log Q_{K, Z}\left(e^{-s}\right)$.

Proof. For each $p \in \mathscr{H}\left(\mathbb{C}^{n+1}\right)$ with $\langle p\rangle \leq 1,\langle p\rangle_{K} \leq e^{-s}$, we see that

$$
\log \langle p(Z)\rangle \in \mathrm{PSH}_{\omega}\left(\mathbb{P}^{n}\right), \quad \log \langle p\rangle \leq 0, \log \langle p\rangle_{K} \leq-s
$$

Thus $\log Q_{K, Z}\left(e^{-s}\right) \leq \sigma(Z, s, K)$.

In order to obtain the reversed inequality, it suffices to show that $\varphi(Z) \leq \log Q_{K, Z}\left(e^{-s}\right)$ for each $\varphi \in \operatorname{PSH}_{\omega}\left(\mathbb{P}^{n}\right)$ with $\varphi \leq-s \chi_{K}$. Let $\varphi$ be such a function with $\varphi(Z)>\sigma(Z, s, K)-\varepsilon$ for arbitrary $\varepsilon>0$. By Proposition 2.1.17, there exists a sequence $\left\{\varphi_{j}\right\}$ in $\left.C^{\infty} \cap \mathrm{PSH}_{\omega}\left(\mathbb{P}^{n}\right)\right\}$ such that $\varphi_{j}>-\infty$ and $\varphi_{j}$ decreases towards $\varphi$. In view of Proposition 2.1.15 applied in $\mathbb{P}^{n}$ and in the open set $\Omega=\left\{Z \in \mathbb{P}^{n}: \varphi(Z)<-s+\varepsilon\right\}$, we have $\varphi_{j} \leq \varepsilon$ on $\mathbb{P}^{n}$ and $\varphi_{j} \leq-s+2 \varepsilon$ on $K$ for all sufficiently large $j$. And since

$$
\varphi_{j}(Z)-2 \varepsilon \geq \varphi(Z)-2 \varepsilon>\sigma(Z, s, K)-3 \varepsilon
$$

it follows from Lemma 4.1.2 that

$$
\varphi_{j}(Z)-2 \varepsilon=\sup \left\{\log \langle p(Z)\rangle: p \in \mathscr{H}\left(\mathbb{C}^{n+1}\right), \log \langle p\rangle \leq \varphi_{j}-2 \varepsilon \text { on } \mathbb{P}^{n}\right\}, \quad Z \in \mathbb{P}^{n}
$$

Thus there exists a $p \in \mathscr{H}\left(\mathbb{C}^{n+1}\right)$ with $\langle p\rangle \leq e^{-\varepsilon}$ on $\mathbb{P}^{n}$ and $\langle p\rangle \leq e^{-s}$ on $K$ such that

$$
\log \langle p(Z)\rangle>\varphi_{j}(Z)-2 \varepsilon-\varepsilon>\sigma(Z, s, K)-4 \varepsilon
$$

Therefore

$$
\log Q_{K, Z}\left(e^{-s}\right)>\log \langle p(Z)\rangle>\sigma(Z, s, K)-4 \varepsilon
$$

Letting $\varepsilon \rightarrow 0$ yields that $\log Q_{K, Z}\left(e^{-s}\right) \geq \sigma(Z, s, K)$, which completes the proof. 
Remark. Proposition 4.1.3 establishes a useful formula $\sigma(Z, s, K)=\log Q_{K, Z}\left(e^{-s}\right)$ which is motivated by [13, Proposition 4.2].

Proposition 4.1.4. Let $K$ be a compact set in $\mathbb{P}^{n}$ and let $X \in \mathbb{P}^{n} \backslash K$. Then $K$ has Property $J$ with respect to $X$ if and only if $\sigma(X, s, K)=0$ for each $s>0$.

Proof. Fix $s>0$. Suppose that $K$ has Property $J$ with respect to $X$. Then there is a sequence $\left\{p_{j}\right\} \subset \mathscr{H}\left(\mathbb{C}^{n+1}\right)$ and $0<\eta<1$ such that

$$
\left\langle p_{j}(X)\right\rangle>\eta, \quad\left\langle p_{j}\right\rangle \leq 1, \quad \lim _{j \rightarrow \infty}\left\langle p_{j}\right\rangle_{K}=0,
$$

which implies that $Q_{K, X}\left(e^{-s}\right)>\eta$. By Proposition 4.1.3 and Proposition 3.1.11, $\sigma(X, s, K)=$ 0 for each $s>0$.

Conversely, given $0<\eta<1$, assume that $\sigma(X, s, K)=0$ for each $s>0$. Then we have $\sigma(X, j \log 2, K)=0$ for each $j \in \mathbb{N}$. By Proposition 4.1.3, $Q_{K, X}\left(2^{-j}\right)=1$. It follows that there exists a sequence $\left\{p_{j}\right\} \subset \mathscr{H}\left(\mathbb{C}^{n+1}\right)$ with $\left\langle p_{j}\right\rangle \leq 1$ and $\left\langle p_{j}\right\rangle_{K} \leq 2^{-j}$ such that

$$
\left\langle p_{j}(X)\right\rangle>Q_{K, X}\left(2^{-j}\right)-(1-\eta)=\eta .
$$

Thus

$$
\left\langle p_{j}(X)\right\rangle>\eta, \quad\left\langle p_{j}\right\rangle \leq 1, \quad \lim _{j \rightarrow \infty}\left\langle p_{j}\right\rangle_{K}=0,
$$

which implies that $K$ has Property $J$ with respect to $X$.

Corollary 4.1.5. Let $K$ be a compact set of $\mathbb{P}^{n}$ and let $X \in \mathbb{P}^{n} \backslash K$. Then $K$ has Property $J$ with respect to $X$ if and only if for each $0<\eta<1$ and each $\varepsilon>0$, there is a polynomial $q \in \mathscr{H}\left(\mathbb{C}^{n+1}\right)$ such that

$$
\langle q(X)\rangle>\eta, \quad\langle q\rangle \leq 1, \quad\langle q\rangle_{K} \leq \varepsilon .
$$

Proof. Let $0<\eta<1$ and $\varepsilon>0$ be given. By Proposition 4.1.3 and Proposition 4.1.4, $K$ has Property $\mathrm{J}$ with respect to $X$ if and only if $Q_{K, X}(\varepsilon)=1$ for each $\varepsilon$ which means that there exists a $q \in \mathscr{H}\left(\mathbb{C}^{n+1}\right)$ with $\langle q\rangle \leq 1$ and $\langle q\rangle_{K} \leq \varepsilon$ such that

$$
\langle q(X)\rangle>Q_{K, X}(\varepsilon)-(1-\eta)=\eta,
$$

as required. 


\subsection{Pluripolar hulls in terms of homogeneous polynomials}

Consider a compact pluripolar set $K \subset \mathbb{P}^{n}$, by Proposition 4.1.3,

$$
K_{\mu, \beta}=\left\{Z \in \mathbb{P}^{n}: Q_{K, Z}(t) \leq e^{\beta} t^{1 / \mu}, 0<t \leq 1\right\}
$$

or, equivalently,

$$
K_{\mu, \beta}=\left\{Z \in \mathbb{P}^{n}:\langle p(Z)\rangle \leq e^{\beta} t^{1 / \mu} \text { if } t>0, p \in \mathscr{H}\left(\mathbb{C}^{n+1}\right),\langle p\rangle \leq 1,\langle p\rangle_{K} \leq t\right\} .
$$

In order to obtain a characterization of the pluripolar hull $K^{*}$ in terms of homogeneous polynomials, we firstly rewrite $K_{\mu, \beta}$ as the set in the following Proposition.

Proposition 4.2.1. Let $K$ be a compact pluripolar set $K \subset \mathbb{P}^{n}$ and let $\mu, \beta>0$. Then

$$
K_{\mu, \beta}=\left\{Z \in \mathbb{P}^{n}:\langle p(Z)\rangle \leq e^{\beta}\langle p\rangle^{1-(1 / \mu)}\langle p\rangle_{K}^{1 / \mu} \text { for } p \in \mathscr{H}\left(\mathbb{C}^{n+1}\right)\right\}
$$

Proof. For convenience, let us denote by $L$ the set on the right side of (4.3). We see immediately that $L \subset K_{\mu, \beta}$. Conversely, assume that $p \in \mathscr{H}\left(\mathbb{C}^{n+1}\right)$ is a nonzero homogeneous polynomial and assume $Z \in K_{\mu, \beta}$. Setting $\langle p\rangle=M$ and $\langle p\rangle_{K} / M=t$, we obtain that $\langle p / M\rangle=1$ and $\langle p / M\rangle_{K}=t$, and hence $\langle p(Z) / M\rangle \leq e^{\beta} t^{1 / \mu}=e^{\beta}\langle p / M\rangle_{K}^{1 / \mu}$. Thus

$$
\langle p(Z)\rangle \leq e^{\beta} M^{1-(1 / \mu)}\langle p\rangle_{K}^{1 / \mu}=e^{\beta}\langle p\rangle^{1-(1 / \mu)}\langle p\rangle_{K}^{1 / \mu},
$$

which implies that $Z \in L$. We have proved that $K_{\mu, \beta} \subset L$, as desired.

Remark. By Theorem 3.2.3 and (4.3), the pluripolar hull $K^{*}$ may be given in terms of homogeneous polynomials as

$$
K^{*}=\cup_{m=1}^{\infty}\left\{Z \in \mathbb{P}^{n}:\langle p(Z)\rangle \leq e^{m}\langle p\rangle^{1-(1 / m)}\langle p\rangle_{K}^{1 / m} \text { for } p \in \mathscr{H}\left(\mathbb{C}^{n+1}\right)\right\} .
$$

Theorem 4.2.2. For each compact pluripolar set $K$ in $\mathbb{P}^{n}$, the pluripolar hull $K^{*}$ is an $F_{\sigma}$ set.

Proof. By Proposition 4.1.3, for each $m \in \mathbb{N}$,

$$
K^{(m)}=\left\{Z \in \mathbb{P}^{n}: Q_{K, Z}(t) \leq e^{m} t^{1 / m}, \quad 0<t \leq 1\right\},
$$


that is,

$$
K^{(m)}=\cap\left\{Z \in \mathbb{P}^{n}:\langle p(Z)\rangle \leq e^{m} t^{1 / m}\right\}
$$

where the intersection is taken over all $0<t \leq 1$ and all homogeneous polynomials $p \in$ $\mathscr{H}\left(\mathbb{C}^{n+1}\right)$ with $\langle p\rangle \leq 1,\langle p\rangle_{K} \leq t$. Since the set $\left\{Z \in \mathbb{P}^{n}:\langle p(Z)\rangle \leq e^{m} t^{1 / m}\right\}$ is closed for each $p$ and each $t$, we see that $K^{(m)}$ is compact for each $m$. Therefore, by Theorem 3.2.3, $K^{*}$ is $F_{\sigma}$.

Remark. If $K$ is in a bounded domain $D$ in $\mathbb{C}^{n}$, then $K_{D}^{*}$ is $F_{\sigma}$, which follows from Poletsky's Jensen measure theory (see [20]).

Theorem 4.2.3. Let $K$ be a compact pluripolar set of $\mathbb{P}^{n}$. Then the following are equivalent:

(a) K has Property J;

(b) $K$ is a complete pluripolar set;

(c) $\sigma(X, s, K)=0$ for each $s>0$ and each $X \in \mathbb{P}^{n} \backslash K$.

Proof. That $(a) \Leftrightarrow(c)$ follows from Proposition 4.1.4. Now we prove that $(b) \Rightarrow(a)$. In fact, suppose (b) holds. Let $X \in \mathbb{P}^{n} \backslash K$. Given $0<\eta<1$ and $\varepsilon>0$. Since $K$ is a complete pluripolar set, there exists $\varphi \in \operatorname{PSH}_{\omega}\left(\mathbb{P}^{n}\right)$ such that $\varphi \leq 0$ on $\mathbb{P}^{n},\{\varphi=-\infty\}=K$, and $\varphi(X)=\log \eta$. By Proposition 2.1.17, there exists a sequence $\left\{\varphi_{j}\right\} \subset \operatorname{PSH}_{\omega}\left(\mathbb{P}^{n}\right) \cap C^{\infty}$ with $\varphi_{j} \leq 0$ on $\mathbb{P}^{n},-\infty<\varphi_{j}<-\log j$ on $K$ such that $\varphi_{j}(X) \geq \log \eta+\varepsilon / j$. By Lemma 4.1.2 there is a $p_{j} \in \mathscr{H}\left(\mathbb{C}^{n+1}\right)$ with $\left\langle p_{j}\right\rangle \leq e^{\varphi_{j}} \leq 1$, and $\left\langle p_{j}\right\rangle_{K} \leq\left. e^{\varphi_{j}}\right|_{K}<1 / j$ such that

$$
\left\langle p_{j}(X)\right\rangle>\exp \left(\varphi_{j}(X)-\frac{\varepsilon}{j}\right)>\eta
$$

Hence,

$$
\left\langle p_{j}(X)\right\rangle>\eta, \quad\left\langle p_{j}\right\rangle \leq 1, \quad \lim _{j \rightarrow \infty}\left\langle p_{j}\right\rangle_{K} \leq \lim _{j \rightarrow \infty} \frac{1}{j}=0
$$

which implies that $K$ has Property $\mathrm{J}$ with respect to $X \in \mathbb{P}^{n} \backslash K$. Since $X \in \mathbb{P}^{n} \backslash K$ is arbitrary, we conclude that $K$ has Property J. 
Then it suffices to show that $(c) \Rightarrow(b)$. Let $X \in \mathbb{P}^{n} \backslash K$ be arbitrary. Suppose that (c) holds. Then $X \in K^{(0)}$ and hence $X$ does not belong to the pluripolar hull $K^{*}$ of $K$ by Theorem 3.2.1. Thus $K^{*}=K$. Note that $K$, as a compact subset of $\mathbb{P}^{n}$, is both $F_{\sigma}$ and $G_{\delta}$. It follows from Lemma 2.4.2 that $K$ is a complete pluripolar set, which completes the proof. 


\section{CHAPTER 5}

\section{CONVERGENCE SETS}

\subsection{Convergence Sets in $\mathbb{P}^{n}$}

Denote by $\mathbb{C}[[z]]:=\mathbb{C}\left[\left[z_{0}, z_{1}, \ldots, z_{n}\right]\right]$ the ring of formal power series of $n+1$ variables $z_{0}, z_{1}, \ldots, z_{n}$ with complex coefficients. Let $f$ be such a formal power series with

$$
f(z)=f\left(z_{0}, z_{1}, \ldots, z_{n}\right)=\sum a_{\alpha} z^{\alpha} \in \mathbb{C}\left[\left[z_{0}, z_{1}, \ldots, z_{n}\right]\right]
$$

where $\alpha \in \mathbb{N}^{n+1}$. The series $f$ is said to converge if it converges absolutely in a neighborhood of the origin in $\mathbb{C}^{n+1}$. Otherwise we say it diverges. So $f$ converges if and only if there is a number $C>0$ such that $\left|a_{\alpha}\right| \leq C^{|\alpha|+1}$ for each $\alpha \in \mathbb{N}^{n+1}$. When $f$ diverges, $f$ may converge in some directions. The convergence set of $f$, denoted by $\operatorname{Conv}(f)$, is the set of $Z \in \mathbb{P}^{n}$ for which $f_{z}(t):=f\left(z_{0} t, z_{1} t, \ldots, z_{n} t\right)$, as a series of one variable $t$, converges (absolutely in some neighborhood of 0 ) for some (and hence all) $z=\left(z_{0}, z_{1}, \ldots, z_{n}\right) \in \pi^{-1}(Z)$. This is welldefined since, when $z \neq 0, f_{z}(t)$ converges if and only if $f_{c z}(t)$ converges for all $c \in \mathbb{C} \backslash\{0\}$. Thus the series $f$ converges if and only if $\operatorname{Conv}(f)=\mathbb{P}^{n}$.

Definition 5.1.1. A subset $E \subset \mathbb{P}^{n}$ is said to be a convergence set in $\mathbb{P}^{n}$ if $E=\operatorname{Conv}(f)$ for some divergent power series $f$. Equivalently, $E$ is a convergence set if $E \neq \mathbb{P}^{n}$ and $E=\operatorname{Conv}(f)$ for some $f \in \mathbb{C}\left[\left[z_{0}, \ldots, z_{n}\right]\right]$.

Denote by $\operatorname{Conv}\left(\mathbb{P}^{n}\right)$ the collection of all convergence sets in $\mathbb{P}^{n}$ :

$$
\operatorname{Conv}\left(\mathbb{P}^{n}\right):=\left\{\operatorname{Conv}(f): f \in \mathbb{C}\left[\left[z_{0}, z_{1}, \ldots, z_{n}\right]\right], f \text { diverges }\right\}
$$

Let $f \in \mathbb{C}[[z]]$ be a divergent series. Since

$$
f_{z}(t):=f\left(z_{0} t, z_{1} t, \ldots, z_{n} t\right)=\sum_{j=1}^{\infty} p_{j}(z) t^{j}, \quad p_{j} \in \mathscr{H}_{j}\left(\mathbb{C}^{n+1}\right),
$$

we see that

$$
\operatorname{Conv}(f)=\left\{Z \in \mathbb{P}^{n}: \sup _{j}\left\langle p_{j}(Z)\right\rangle<\infty\right\}
$$


In fact, we have the following lemma (see [27]).

Lemma 5.1.2. Suppose that $E \varsubsetneqq \mathbb{P}^{n}$. Then $E \in \operatorname{Conv}\left(\mathbb{P}^{n}\right)$ if and only if there exists a countable family $\mathscr{F}$ of non-constant homogeneous polynomials in $\mathscr{H}\left(\mathbb{C}^{n+1}\right)$ such that

$$
E=\left\{Z \in \mathbb{P}^{n}: \sup _{p \in \mathscr{F}}\langle p(Z)\rangle<\infty\right\}
$$

Proposition 5.1.3. If $E_{j} \in \operatorname{Conv}\left(\mathbb{P}^{n}\right)$ for each $j=1,2, \ldots, m$, then $\bigcap_{j=1}^{m} E_{j} \in \operatorname{Conv}\left(\mathbb{P}^{n}\right)$.

Proof. Suppose that $E_{1}, E_{2}, \ldots, E_{m} \in \operatorname{Conv}\left(\mathbb{P}^{n}\right)$. Then Lemma 5.1 .2 implies that there are countable families $\mathscr{F}_{1}, \mathscr{F}_{2}, \ldots, \mathscr{F}_{m}$, of non-constant homogeneous polynomials such that

$$
E_{j}=\left\{Z \in \mathbb{P}^{n}: \sup _{p \in \mathscr{F}_{j}}\langle p(Z)\rangle<\infty\right\}, \quad j=1,2, \ldots, m
$$

It follows that

$$
\cap_{j=1}^{m} E_{j}=\left\{Z \in \mathbb{P}^{n}: \sup _{p \in \cup_{j=1}^{m} \mathscr{F}_{j}}\langle p(Z)\rangle<\infty\right\} .
$$

Therefore, $\cap_{j=1}^{m} E_{j} \in \operatorname{Conv}\left(\mathbb{P}^{n}\right)$.

We do not know whether the union of a finite number of convergence sets is necessarily a convergence set or not.

Proposition 5.1.4. Let $K$ be a compact pluripolar set in $\mathbb{P}^{n}$. Then $K^{(0)}=K^{(m)(0)}$ for $m \geq 1$.

Proof. Since $K \subset K^{(m)}$, by Proposition 2.4.3 and Theorem 3.2.3 we have

$$
K^{*} \subset\left(K^{(m)}\right)^{*} \subset K^{* *}=K^{*}
$$

It follows that $K^{*}=\left(K^{(m)}\right)^{*}$. By Theorem 3.2.1 we obtain

$$
K^{(0)}=K^{(m)(0)}
$$

as required.

Theorem 5.1.5. Let $\left\{K_{j}\right\}$ be a sequence of compact pluripolar sets in $\mathbb{P}^{n}$. Then $K:=$ $\cup_{j=1}^{\infty} K_{j}^{*}$ is a convergence set. 
Proof. Let $E_{k}=\cup_{j=1}^{k} K_{j}^{(k)}$. Then $E_{k}$ is a compact pluripolar set in $\mathbb{P}^{n}$ and by Theorem 3.2.3,

$$
\begin{aligned}
K & =\cup_{j=1}^{\infty} K_{j}^{*} \\
& =\cup_{j=1}^{\infty} \cup_{m=1}^{\infty} K_{j}^{(m)} \\
& =\cup_{k=1}^{\infty}\left(\cup_{j=1}^{k} \cup_{m=1}^{k} K_{j}^{(m)}\right) \\
& =\cup_{k=1}^{\infty}\left(\cup_{j=1}^{k} K_{j}^{(k)}\right) \\
& =\cup_{k=1}^{\infty} E_{k} .
\end{aligned}
$$

Assume that $X \in \mathbb{P}^{n} \backslash K$ and $k \geq 1$. Then $X \notin K_{j}^{*}$ and hence $X \in K_{j}^{(0)}$ for each $j$. By Corollary 3.2.2 and Proposition 5.1.4, $X \in E_{k}^{(0)}=\cap_{j=1}^{k} K_{j}^{(0)}$, which implies that $E_{k}$ has Property $\mathrm{J}$ with respect to $X$ by Proposition 4.1.4. For given $0<\eta<1$, by Corollary 4.1.5 there is a homogeneous polynomial $p \in \mathscr{H}\left(\mathbb{C}^{n+1}\right)$ such that

$$
\langle p(X)\rangle>\eta, \quad\langle p\rangle \leq 1, \quad \text { and } \quad\langle p\rangle_{E_{k}}<k^{-1 / \beta},
$$

where $\beta=a / b<1$ is a rational number with $a, b$ being coprime positive integers so that $(\eta / k)^{\beta}>1 / 2$.

Let $d=\operatorname{deg} p$ and $x \in \pi^{-1}(X)$. Define $q \in \mathscr{H}\left(\mathbb{C}^{n+1}\right)$ by

$$
q(y)=\left(k y \cdot \frac{\bar{x}}{|x|}\right)^{d}=\left[k|x|^{-1}\left(y_{0} \bar{x}_{0}+\cdots+y_{n} \bar{x}_{n}\right)\right]^{d},
$$

we have $\operatorname{deg} q=\operatorname{deg} p=d$, and

$$
\begin{aligned}
\langle q\rangle & =\sup _{Z \in \mathbb{P}^{n}}\langle q(Z)\rangle \\
& =\sup _{0 \neq z \in \mathbb{C}^{n+1}} \frac{|q(z)|^{1 / \operatorname{deg} q}}{|z|} \\
& =\sup _{0 \neq z \in \mathbb{C}^{n+1}} \frac{(k /|x|)\left|z_{0} \bar{x}_{0}+\cdots+z_{n} \bar{x}_{n}\right|}{|z|} \\
& =\frac{k}{|x|} \sup _{0 \neq z \in \mathbb{C}^{n+1}} \frac{\left|z_{0} \bar{x}_{0}+\cdots+z_{n} \bar{x}_{n}\right|}{|z|} \\
& =\frac{k}{|x|} \cdot|x|=k .
\end{aligned}
$$


The penultimate step is by the Cauchy-Schwarz inequality. It follows that $\langle q\rangle=k=\langle q(X)\rangle$. Let $h:=p^{a} q^{b-a}$. Then $h \in \mathscr{H}\left(\mathbb{C}^{n+1}\right)$ and $\operatorname{deg} h=\operatorname{deg}\left(p^{a} q^{b-a}\right)=b d$. For each $Z \in \mathbb{P}^{n}$,

$$
\begin{aligned}
\langle h(Z)\rangle & =\frac{|h(Z)|^{1 /(b d)}}{|Z|} \\
& =\frac{\left|p^{a}(Z) q^{b-a}(Z)\right|^{1 /(b d)}}{|Z|} \\
& =\frac{|p(Z)|^{a /(b d)}}{|Z|^{a / b}} \cdot \frac{|q(Z)|^{(b-a) /(b d)}}{|Z|^{(b-a) / b}} \\
& =\left(\frac{|p(Z)|^{1 / d}}{|Z|}\right)^{a / b}\left(\frac{|q(Z)|^{1 / d}}{|Z|}\right)^{(b-a) / b} \\
& =\langle p(Z)\rangle^{\beta}\langle q(Z)\rangle^{1-\beta} .
\end{aligned}
$$

Hence,

$$
\begin{aligned}
\langle h\rangle & =\sup _{Z \in \mathbb{P}^{n}}\langle h(Z)\rangle=\sup _{Z \in \mathbb{P}^{n}}\langle p(Z)\rangle^{\beta}\langle q(Z)\rangle^{1-\beta} \leq 1^{\beta} \cdot k^{1-\beta} \leq k, \\
\langle h\rangle_{E_{k}} & =\sup _{Y \in E_{k}}\langle h(Y)\rangle=\sup _{Y \in E_{k}}\langle p(Y)\rangle^{\beta}\langle q(Y)\rangle^{1-\beta} \leq k^{-1} \cdot k^{1-\beta}=k^{-\beta}<1, \\
\langle h(X)\rangle & =\langle p(X)\rangle^{\beta}\langle q(X)\rangle^{1-\beta}>\eta^{\beta} \cdot k^{1-\beta}=(\eta / k)^{\beta} \cdot k>k / 2 .
\end{aligned}
$$

To summarize, there is an $h_{X} \in \mathscr{H}\left(\mathbb{C}^{n+1}\right)$ such that

$$
\left\langle h_{X}\right\rangle_{E_{k}}<1, \quad\left\langle h_{X}\right\rangle \leq k, \text { and }\left\langle h_{X}(X)\right\rangle>k / 2
$$

Let $U_{X}:=\left\{Z \in \mathbb{P}^{n}:\left\langle h_{X}(Z)\right\rangle>k / 2\right\}$. Then $U_{X}$ is a neighborhood of $X$. The open cover $\left\{U_{X}: X \in \mathbb{P}^{n} \backslash K\right\}$ of $\mathbb{P}^{n} \backslash K$ contains a countable subcover $\left\{U_{X_{j}}: j=1,2, \ldots\right\}$. Put $h_{k j}=h_{X_{j}}$. We see that the sequence $\left\{h_{k j}\right\}_{j}$ satisfies

(i) $\left\langle h_{k j}\right\rangle_{E_{k}}<1$,

(ii) $\left\langle h_{k j}\right\rangle \leq k$,

(iii) $\cup_{j=1}^{\infty}\left\{Z \in \mathbb{P}^{n}:\left\langle h_{k j}(Z)\right\rangle>k / 2\right\} \supset \mathbb{P}^{n} \backslash K$.

Enumerate the countable sequence $\left\{h_{k j}\right\}$ to obtain a single sequence $\left\{p_{\ell}\right\}$ and choose $m_{\ell}$ so that $\operatorname{deg} p_{\ell}^{m_{\ell}}$ is increasing. Set $q_{\ell}:=p_{\ell}^{m_{l}}$ and let $f:=\sum q_{\ell}$. Then the convergence set of $f$ is given by

$$
\operatorname{Conv}(f)=\left\{Z \in \mathbb{P}^{n}: \sup _{\ell}\left\langle q_{\ell}(Z)\right\rangle<\infty\right\}=\left\{Z \in \mathbb{P}^{n}: \sup _{k, j}\left\langle h_{k j}(Z)\right\rangle<\infty\right\}
$$


Suppose that $Z \in K$. Then there is an $k_{0} \geq 2$ such that $Z \in E_{k_{0}}$. For $k \geq k_{0}$, $\left\langle h_{k j}(Z)\right\rangle \leq 1$ and for $k<k_{0},\left\langle h_{k j}(Z)\right\rangle \leq k \leq k_{0}-1$. Hence $\sup _{k, j}\left\langle h_{k j}(Z)\right\rangle \leq k_{0}-1<\infty$, which implies $K \subset \operatorname{Conv}(f)$.

Suppose that $Z \in \mathbb{P}^{n} \backslash K$. Then by (iii), $\sup _{j}\left\langle h_{k j}(Z)\right\rangle \geq k / 2$. Hence, $\sup _{k, j}\left\langle h_{k j}(Z)\right\rangle=\infty$, that is, $Z \in \mathbb{P}^{n} \backslash\left\{Z \in \mathbb{P}^{n}: \sup _{k, j}\left\langle h_{k j}(Z)\right\rangle<\infty\right\}$. Thus $\operatorname{Conv}(f) \subset K$. Therefore,

$$
K=\operatorname{Conv}(f) .
$$

By Lemma 5.1.2, $K$ is a convergence set in $\mathbb{P}^{n}$.

Remark. In the proof of Theroem 5.1.5, we rewrite the union of the pluripolar hulls of a countable collection of compact pluripolar sets as a union $\cup_{k=1}^{\infty} E_{k}$ by (5.1) and obtain that $E_{k}$ has Property J with respect to $X \in \mathbb{P}^{n} \backslash\left(\cup_{j=1}^{\infty} K_{j}^{*}\right)$ for each $k$. But in general $E_{k}$ does not have Property J (i.e., $E_{k}$ does not have Property $J$ with respect to every point in $\mathbb{P}^{n} \backslash E_{k}$ ) and hence it is not a complete pluripolar set. Moreover, $K_{j}^{*}$ may be noncompact, and may not be a complete pluripolar set. Therefore, our main result, Theroem 5.1.5, is more general than Theorem 4.18 in [21].

\subsection{Convergence Sets in $\mathbb{C}^{n}$}

Let $\mathbb{C}\{z\}$ be the ring of all power series $g(z) \in \mathbb{C}[[z]]$, where $z=\left(z_{1}, \ldots, z_{n}\right)$, that are absolutely convergent in a neighborhood of the origin in $\mathbb{C}^{n}$.

Let $\Lambda_{n}$ be the set of series $g(t, z)=\sum_{k=0}^{\infty} P_{k}(z) t^{k} \in \mathbb{C}\left[z_{1}, z_{2}, \ldots, z_{n}\right][[t]]$ with $P_{k} \in$ $\mathscr{P}_{k}\left(\mathbb{C}^{n}\right)$. Since we have the convention that the degree of the zero polynomial is -1 , the polynomials $P_{k}$ are allowed to be 0 . For $g \in \Lambda_{n}$, let $\operatorname{Conv}(g)$ be the set of $z \in \mathbb{C}^{n}$ for which $g(t, z)$ converges as a series of one variable $t$ :

$$
\operatorname{Conv}(g):=\left\{z \in \mathbb{C}^{n}: g(t, z) \in \mathbb{C}\{t\}\right\} .
$$

By Hartog's theorem (see [12]), $\operatorname{Conv}(g)=\mathbb{C}^{n}$ if and only if $g \in \mathbb{C}\left\{t, z_{1}, \ldots, z_{n}\right\}$, where $\mathbb{C}\left\{t, z_{1}, \ldots, z_{n}\right\}$ denote the set of convergent series in $n+1$ variables $t, z_{1}, \ldots, z_{n}$.

Definition 5.2.1. A subset $F$ of $\mathbb{C}^{n}$ is said to be a convergence set in $\mathbb{C}^{n}$ if $F=\operatorname{Conv}(g)$ for some divergent power series $g \in \Lambda_{n}$. 
Denote by $\operatorname{Conv}\left(\mathbb{C}^{n}\right)$ the collection of all convergence sets in $\mathbb{C}^{n}$ :

$$
\operatorname{Conv}\left(\mathbb{C}^{n}\right):=\left\{\operatorname{Conv}(g): g \in \Lambda_{n}, g \text { diverges }\right\}
$$

Note that if $z \in \operatorname{Conv}(g)$ for a divergent series $g$, it does not follow that $\lambda z \in \operatorname{Conv}(g)$ for each $\lambda \in \mathbb{C}$, and therefore $\pi(\operatorname{Conv}(g))$ in general is not a convergence set in $\mathbb{P}^{n-1}$.

For $P \in \mathscr{P}_{k}\left(\mathbb{C}^{n}\right)$ with $k>0$ and a subset $F$ of $\mathbb{C}^{n}$, we set

$$
\langle P(z)\rangle_{k}:=\frac{|P(z)|^{1 / k}}{\sqrt{1+|z|^{2}}}, \quad\langle P\rangle_{k, F}=\sup _{z \in F}\langle P(z)\rangle_{F},\langle P\rangle_{k}=\langle P\rangle_{k, \mathbb{C}^{n}}
$$

Note that if $P \in \mathscr{P}_{k}\left(\mathbb{C}^{n}\right)$ and $m$ is a positive integer, then $\left\langle P^{m}(z)\right\rangle_{k m}=\langle P(z)\rangle_{k}$. Denote by $\mathscr{P}\left(\mathbb{C}^{n}\right)$ the family of polynomials in $n$ variables $z_{1}, \ldots, z_{n}$ with complex coefficients in $\mathbb{C}$.

We have the following lemma which is analogous to Lemma 5.1.2.

Lemma 5.2.2. Suppose that $F \varsubsetneqq \mathbb{C}^{n}$. Then $F \in \operatorname{Conv}\left(\mathbb{C}^{n}\right)$ if and only if there exists a sequence $\left\{j_{k}\right\} \subset \mathbb{N}$ and a sequence $\left\{P_{k}\right\}$ of polynomials with $P_{k} \in \mathscr{P}_{j_{k}}\left(\mathbb{C}^{n}\right)$ for all $k$ such that

$$
F=\left\{z \in \mathbb{C}^{n}: \sup _{k}\left\langle P_{k}(z)\right\rangle_{j_{k}}<\infty\right\} .
$$

Note that $\iota: \mathbb{C}^{n} \rightarrow \mathbb{P}^{n}$ defined by (3.1) identifies $\mathbb{C}^{n}$ with $\iota\left(\mathbb{C}^{n}\right)=U_{0}$. Define $\gamma: \Lambda_{n} \rightarrow$ $\mathbb{C}\left[\left[z_{0}, \ldots, z_{n}\right]\right]$ by

$$
\gamma\left(\sum_{k=0}^{\infty} P_{k}(z) t^{k}\right)=\sum_{k=0}^{\infty} z_{0}^{k} P_{k}\left(z / z_{0}\right)
$$

Put $p_{k}\left(z_{0}, z\right):=z_{0}^{k} P_{k}\left(z / z_{0}\right)$. Then $p_{k} \in \mathscr{H}_{k}\left(\mathbb{C}^{n+1}\right)$ for $k \geq 0$, and $\left\langle p_{k}(\iota(z))\right\rangle=\left\langle P_{k}(z)\right\rangle_{k}$ for $k \geq 1$. It follows from Lemma 5.1.2 and Lemma 5.2.2 that

$$
\iota(\operatorname{Conv}(g))=U_{0} \cap \operatorname{Conv}(\gamma(g)) .
$$

Consequently, we have the following proposition.

Proposition 5.2.3. Suppose that $F \varsubsetneqq \mathbb{C}^{n}$. Then $F \in \operatorname{Conv}\left(\mathbb{C}^{n}\right)$ if and only if $\iota(F)=U_{0} \cap E$ for some $E \in \operatorname{Conv}\left(\mathbb{P}^{n}\right)$. 
Remark. For a convergence set $F$ in $\mathbb{C}^{n}$, the set $\iota(F)$ may or may not be a convergence set in $\mathbb{P}^{n}$ (see Example 7.2 and 7.3).

Let $W$ be a compact pluripolar subset of $\mathbb{C}^{n}$. Then the pluripolar hull of $W$ is given by

$$
W^{*}=\iota^{-1}\left(U_{0} \cap(\iota(W))^{*}\right) .
$$

It follows that $W^{*}=\cup_{m=1}^{\infty} W^{(m)}$, where

$$
W^{(m)}=\left\{z \in \mathbb{C}^{n}:\langle P(z)\rangle \leq e^{m}\langle P\rangle_{k}^{1-1 / m}\langle P\rangle_{k, W}^{1 / m} \quad \text { for } \quad k \in \mathbb{N}, P \in \mathscr{P}_{k}\left(\mathbb{C}^{n}\right)\right\}
$$

By Proposition 2.4.4 and Theorem 5.1.5, and in view of (5.2), we have

Theorem 5.2.4. The union of the pluripolar hulls of a countable collection of closed pluripolar sets in $\mathbb{C}^{n}$ is a convergence set in $\mathbb{C}^{n}$. 


\section{CHAPTER 6}

\section{CONVERGENCE SETS ON $\Gamma$}

\subsection{Polynomial Hulls and projective hulls}

Definition 6.1.1. The polynomial hull $\tilde{W}$ of a compact set $W \subset \mathbb{C}^{n}$ is the set of all points $z \in \mathbb{C}^{n}$ such that

$$
|P(z)| \leq\|P\|_{W}
$$

for all polynomials $P \in \mathscr{P}\left(\mathbb{C}^{n}\right)$. A compact set $W \in \mathbb{C}^{n}$ is said to be polynomially convex if $\tilde{W}=W$.

Proposition 6.1.2. Let $W_{1}, W_{2}, \cdots, W_{k}$ be pairwise disjoint compact sets in $\mathbb{C}$. Then $\cup_{j=1}^{k} \tilde{W}_{j}$ is the polynomial hull of $\cup_{j=1}^{k} W_{j}$.

Proof. It suffices to show that the polynomial hull of $\cup_{j=1}^{k} W_{j}$ is contained in $\cup_{j=1}^{k} \tilde{W}_{j}$ for the case $k=2$. Suppose that $W_{1}, W_{2}$ are compact sets with $W_{1} \cap W_{2}=\varnothing$. Let $U$ be a bounded connected component of $\mathbb{C} \backslash\left(W_{1} \cup W_{2}\right)$, and let $D=: \partial \tilde{\bar{U}}$ be the boundary of the polynomial hull of the closure of $U$. Then

$$
D \subset \partial \bar{U} \subset \partial U \subset \partial\left(\mathbb{C} \backslash\left(W_{1} \cup W_{2}\right)\right)=\partial\left(W_{1} \cup W_{2}\right) \subset\left(W_{1} \cup W_{2}\right)^{-}=W_{1} \cup W_{2}
$$

Since $D$ is connected, it follows that $D \subset W_{1}$ or $W_{2}$, and hence $U \subset \tilde{D} \subset \tilde{W}_{1} \cup \tilde{W}_{2}$. Therefore, the polynomial hull of $W_{1} \cup W_{2}$ is contained in $\tilde{W}_{1} \cup \tilde{W}_{2}$.

Definition 6.1.3. (see, e.g., [13]) The projective hull $\hat{K}$ of a compact set $K \subset \mathbb{P}^{n}$ is the set of all points $Z \in \mathbb{P}^{n}$ for which there exists a constant $C=C_{Z}>0$ such that

$$
\langle p(Z)\rangle \leq C\langle p\rangle_{K}
$$

for all homogeneous polynomials $p \in \mathscr{H}\left(\mathbb{C}^{n+1}\right)$. A compact set $K \subset \mathbb{P}^{n}$ is said to be projectively convex if $\hat{K}=K$. 
It follows directly from the definition that if $K_{1} \subset K_{2}$ then $\hat{K}_{1} \subset \hat{K}_{2}$. Note that each algebraic variety in $\mathbb{P}^{n}$ is projectively convex. In particular, each finite set is projectively convex (see, e.g., [21]).

Remark. (a) The projective hull of a compact set $K \subset \mathbb{P}^{n}$ defined above is equivalent to that in $[13$, p. 607$]$ because for $k \geq 1$, the set $H^{0}\left(\mathbb{P}^{n}, \mathcal{O}(k)\right)$ of global holomorphic sections of the line bundle $\mathcal{O}(k)$ is canonically identified with the set $\mathscr{H}_{k}\left(\mathbb{C}^{n+1}\right)$ of homogeneous polynomials of degree $k$. We caution that the above definition for projective hulls differs substantially from that of $\hat{X}_{h}$ (the $h$ in the subscript stands for "homogeneous") in $[28$, p. 116] in that the above definition contains a constant $C$ while the definition in [28] requires that $C=1$. For instance, for $X=\{(z, 0): z \in \mathbb{C},|z| \leq 1\} \subset \mathbb{C}^{2} \subset \mathbb{P}^{2}$, the projective hull $\hat{X}$ (in $\mathbb{C}^{2}$ ) is $\{(z, 0): z \in \mathbb{C}\}$, while the $\hat{X}_{h}$ in [28] equals $X$.

(b) By (4.3) we have

$$
K_{1, \beta}=\left\{Z \in \mathbb{P}^{n}:\langle p(Z)\rangle \leq e^{\beta}\langle p\rangle_{K} \text { for } p \in \mathscr{H}\left(\mathbb{C}^{n+1}\right)\right\} .
$$

It follows that $\hat{K}=K_{1, \infty}=\cup_{\beta>0} K_{1, \beta}$. Furthermore, by Theorem 3.2.3 we obtain that $\hat{K} \subset K^{*}$, which was also obtained in [21, Proposition 3.6] with a different proof.

The following two useful results, which were proved by using a theorem in [11], can be found in [13].

Proposition 6.1.4. (see [13, Corollary 4.4]) Let $K \subset \mathbb{P}^{n}$ be a compact subset. Then the following are equivalent:

(a) $K$ is pluripolar.

(b) $\hat{K}$ is pluripolar.

(c) $\hat{K} \neq \mathbb{P}^{n}$.

Proposition 6.1.5. (see [13, Proposition 9.1]) Let $K$ be an irreducible algebraic subvariety of $\mathbb{P}^{n}$, and let $E \subset K$ be nonpluripolar in $K$. Then $\hat{E}=K$.

Proposition 6.1.6. Let $K:=\{p=0\} \subset \mathbb{P}^{n}$ be an algebraic variety, where $p \in \mathscr{H}\left(\mathbb{C}^{n+1}\right)$. For each convergence set $E$ in $\mathbb{P}^{n}$, either $K \cap E$ is pluripolar in $K$, or $K \subset E$. 
Proof. By [21, Theorem 4.6] there exists an ascending sequence $\left\{K_{j}\right\}$ of compact pluripolar sets such that $E=\cup K_{j}=\cup \hat{K}_{j}$. Then either $K \cap K_{j}$ is pluripolar in $K$ for each $j$, or $K \cap K_{j}$ is nonpluripolar in $K$ for some $j$. The former implies that $K \cap E=\cup_{j}\left(K \cap K_{j}\right)$ is pluripolar in $K$, while by Proposition 6.1.5 the latter implies that $\left(K \cap K_{j}\right)^{\wedge}=K$ for such $j$ and hence $K \subset E$. This completes the proof.

By [13, Theorem 9.2], which depends on a deep theorem in [25], we have the following Lemma.

Lemma 6.1.7. Let $W$ be a compact subset in $\mathbb{C}$, the set $\Gamma(W)$ is projectively convex in $\mathbb{P}^{2}$ if and only if $W$ is polynomially convex in $\mathbb{C}$.

\subsection{Convergence Sets on $\Gamma$}

We fix a non-polynomial entire holomorphic function $\psi(z)$ on the complex plane, say $\psi(z)=e^{z}$. Denote by $\Gamma$ the image under the embedding $\mathbb{C}^{2} \rightarrow \mathbb{P}^{2}$ of the graph of $\psi$ :

$$
\Gamma:=\left\{[1: z: \psi(z)] \in \mathbb{P}^{2}: z \in \mathbb{C}\right\}
$$

For a subset $S$ of $\mathbb{C}$, let $\Gamma(S):=\left\{[1: z: \psi(z)] \in \mathbb{P}^{2}: z \in S\right\}$. Obviously, every subset of $\Gamma$ is $\Gamma(S)$ for some $S \subset \mathbb{C}$.

In this section we obtain a characterization of subsets of $\Gamma$ which are convergence sets in $\mathbb{P}^{2}$. Such a set is called a convergence set on $\Gamma$. Observe that a convergence set on $\Gamma$ is necessarily a convergence set in $\mathbb{C}^{2}$. The main idea of this section is motivated by [13,

Theorem 9.2] and some tools we use here to obtain the main result of this section come from $[2]$.

By [21, Lemma 4.7] we have the following Proposition.

Proposition 6.2.1. Let $\left\{K_{j}\right\}$ be an ascending sequence of projectively convex, compact, pluripolar sets in $\mathbb{P}^{n}$ and let $K:=\cup_{j=1}^{\infty} K_{j}$. Let $\left\{U_{j}\right\}$ be a sequence of open sets such that $K_{j} \subset U_{j}$ and $\cup_{m=1}^{\infty} \cap_{j=m}^{\infty} U_{j} \subset K$. Then $K$ is a convergence set in $\mathbb{P}^{n}$.

Corollary 6.2.2. Let $\left\{K_{j}\right\}$ be an ascending sequence of projectively convex, compact, pluripolar sets in $\mathbb{P}^{n}$ such that $K:=\cup_{j=1}^{\infty} K_{j}$ is $G_{\delta}$. Then $K \in \operatorname{Conv}\left(\mathbb{P}^{n}\right)$. 
For $r>0$ and $S \subset \mathbb{C}$, we define the $r$-neighborhood $N_{r}(S)$ of $S$ by

$$
N_{r}(S)=\{z \in \mathbb{C}:|z-s|<r \text { for some } s \in S\}
$$

and denote $d(z, S)$ the Euclidean distance between the point $z$ and the set $S$.

Proposition 6.2.3. Let $\left\{W_{j}\right\}$ be a sequence of compact, polynomially convex sets in $\mathbb{C}$ and let $F=\cup_{j=1}^{\infty} W_{j}$. Then there exists an ascending sequence $\left\{F_{k}\right\}$ of polynomially convex compact sets in $\mathbb{C}$ such that

$$
F=\cup_{k=1}^{\infty} F_{k}
$$

and such that the neighborhoods $V_{k}:=N_{1 /(3 k)}\left(F_{k}\right)$ satisfy

$$
\cap_{k=m}^{\infty} V_{k} \subset F
$$

for each integer $m \geq 1$.

Proof. For $1 \leq j \leq k$, set

$$
L_{k j}=W_{j} \backslash \cup_{\ell=1}^{j-1} N_{1 / k}\left(W_{\ell}\right), \text { and } K_{k j}=\tilde{L}_{k j} \text {. }
$$

Then we have

$$
L_{k \ell} \cap L_{k j}=\varnothing \quad \text { for } 1 \leq \ell<j \leq k
$$

and

$$
L_{k j} \subset L_{k+1, j}
$$

Let $G_{k}=\cup_{j=1}^{k} L_{k j}$, and $F_{k}=\cup_{j=1}^{k} K_{k j}$. Then $G_{k} \subset G_{k+1}$ and by Proposition 6.1.2, $F_{k}=\tilde{G}_{k}$ for each $k$. Hence $F_{k} \subset F_{k+1}$ and $F_{k}$, as the polynomial hull of $G_{k}$, is polynomially convex.

Suppose that $z \in F$. Let $j$ be the least integer such that $z \in W_{j}$. If $j=1$, then $z \in W_{1}=F_{1}$. Assume $j>1$ and choose $k \geq j$ so that $d\left(z, \cup_{i=1}^{j-1} W_{i}\right)>1 / k$. Then

$$
z \in L_{k j} \subset K_{k j} \subset F_{k}
$$


which implies $F \subset \cup_{k=1}^{\infty} F_{k}$.

Conversely, since

$$
F_{k}=\cup_{j=1}^{k} K_{k j} \subset \cup_{j=1}^{k} W_{j} \subset F,
$$

we see that $\cup_{k=1}^{\infty} F_{k} \subset F$. This completes the proof of (6.1).

We now prove (6.2). Fixing an integer $m \geq 1$, we will show that the assumption $\left(\cap_{k=m}^{\infty} V_{k}\right) \backslash F \neq \varnothing$ leads to a contradiction. Assume that $z \in\left(\cap_{k=m}^{\infty} V_{k}\right) \backslash F$. We now prove by induction that

$$
z \in N_{1 /(3 k)}\left(\cup_{j=1}^{m} K_{k j}\right), \text { for } k \geq m
$$

When $k=m$,

$$
z \in V_{m}=N_{1 /(3 m)}\left(\cup_{j=1}^{m} K_{m j}\right)
$$

We assume that $M>m$ and (6.3) holds for $k=M-1$, that is,

$$
z \in N_{1 /(3 M-3)}\left(\cup_{j=1}^{m} K_{M-1, j}\right)
$$

Set

$$
Q=\cup_{j=m+1}^{M} K_{M j}, \quad R=Q \cap\left(\cup_{j=1}^{m} K_{M j}\right), \quad S=\left(\cup_{j=1}^{m} K_{M j}\right) \backslash R .
$$

Then $Q \cap S=\varnothing$, and $Q \cup S=F_{M}$.

Consider a point $w \in R$. There exist $i, \ell$ with $1 \leq i \leq m<\ell \leq M$ such that $w \in$ $K_{M i} \cap K_{M \ell}$. By the construction of $L_{M \ell}$ and $K_{M \ell}, w \in K_{M \ell} \backslash L_{M \ell}$ and hence $w$ belongs to a bounded connected component $U$ of the complement $L_{M \ell}^{c}$ of $L_{M \ell}$. The connected open set $N_{1 / M}(w)$ is contained in $L_{M \ell}^{c}$, so it is contained in some connected component of $L_{M \ell}^{c}$; since $w \in U$, we obtain that $N_{1 / M}(w) \subset U \subset K_{M \ell}$. Thus,

$$
N_{1 / M}(R) \subset Q
$$

Now we consider a point $w \in S$ and a $j$ with $m+1 \leq j \leq M$. Since $w \in N_{1 / M}\left(\cup_{i=1}^{m} W_{i}\right)$ and since $w \notin K_{M j}$, we see that $w$ belongs to the unbounded connected component $V$ of 
$L_{M j}^{c}$. The connected open set $N_{1 / M}(w)$ is contained in $L_{M j}^{c}$, so it must be contained in $V$. It follows that $N_{1 / M}(w) \cap K_{M j}=\varnothing$. Therefore,

$$
N_{1 / M}(S) \cap Q=\varnothing .
$$

By the induction hypothesis, we have

$$
\left.z \in N_{1 /(3 M-3)}\left(\cup_{j=1}^{m} K_{M-1, j}\right) \subset N_{1 /(3 M-3)}\left(\cup_{j=1}^{m} K_{M j}\right)=N_{1 /(3 M-3)}(S \cup R)\right),
$$

and hence

$$
z \in N_{1 /(3 M-3)}(S) \cup N_{1 /(3 M-3)}(R) .
$$

However, since $N_{1 /(3 M-3)}(R) \subset N_{1 / M}(R) \subset Q \subset F$ by (6.6), we see that

$$
z \notin N_{1 /(3 M-3)}(R) .
$$

Now (6.7) and (6.8) imply that

$$
z \in N_{1 /(3 M-3)}(S)
$$

Making use of (6.6) and (6.9), we obtain that

$$
\begin{aligned}
d(z, Q) & \geq d(S, Q)-d(z, S) \\
& \geq \frac{1}{M}-\frac{1}{3 M-3} \\
& \geq \frac{1}{M}-\frac{2}{3 M} \\
& =\frac{1}{3 M},
\end{aligned}
$$

and hence $z \notin N_{1 /(3 M)}(Q)$. This, together with $z \in V_{M}=N_{1 /(3 M)}(S \cup Q)$, implies that

$$
z \in N_{1 /(3 M)}(S) \subset N_{1 /(3 M)}\left(\cup_{j=1}^{m} K_{M j}\right) .
$$

This completes the proof of (6.3).

Since $\cup_{j=1}^{m} K_{k j} \subset \cup_{j=1}^{m} W_{j}$, (6.3) implies that

$$
d\left(z, \cup_{j=1}^{m} W_{j}\right)<\frac{1}{3 k}, \text { for each } k \geq m .
$$

Letting $k \rightarrow \infty$ yields that $z \in \cup_{j=1}^{m} W_{j} \subset F$, which is a contradiction, as desired. 
Theorem 6.2.4. $E \subset \Gamma$ is a convergence set if and only if $E=\cup_{j=1}^{\infty} E_{j}$, where $E_{j}$ is compact and projectively convex for each $j$.

Proof. Suppose that $E \subset \Gamma$ is a convergence set and $E=\operatorname{Conv}(f)$ with $f=\sum_{m=1}^{\infty} h_{m}$, where $h_{m} \in \mathscr{H}_{m}\left(\mathbb{C}^{3}\right)$. Then $E=\cup_{j=1}^{\infty} E_{j}$, where

$$
E_{j}:=\Gamma\left(W_{j}\right), \text { with } W_{j}:=\left\{z \in \mathbb{C}:\left\langle h_{m}(1, z, \psi(z))\right\rangle \leq j, \forall m\right\}
$$

Since $W_{j}$ is compact and polynomially convex in $\mathbb{C}$ for each $j$, by Lemma 6.1 .7 , it follows that each $E_{j}$ is compact and projectively convex in $\mathbb{P}^{2}$.

Conversely, suppose that $E=\cup_{j=1}^{\infty} E_{j}$, where $E_{j}$ are compact and projectively convex. For a fixed positive integer $j$, let $W_{j}$ be the subset of $\mathbb{C}$ with $E_{j}=\Gamma\left(W_{j}\right)$. By Lemma 6.1.7, $W_{j}$ is compact and polynomially convex in $\mathbb{C}$. Let $F=\cup_{j=1}^{\infty} W_{j}$. By Proposition 6.2.3, there exists an ascending sequence $\left\{F_{j}\right\}$ of compact and polynomially convex sets such that $F=\cup_{j=1}^{\infty} F_{j}$, and

$$
\cup_{m=1}^{\infty} \cap_{j=m}^{\infty} V_{j} \subset F
$$

where $V_{j}:=N_{1 /(3 j)}\left(F_{j}\right)$. Consequently, there exists a sequence $\left\{U_{j}\right\}$ of open sets with $E_{j} \subset U_{j}$ on $\Gamma$ such that

$$
\cup_{m=1}^{\infty} \cap_{j=m}^{\infty} U_{j} \subset E .
$$

By Proposition 6.2.1, $E \in \operatorname{Conv}\left(\mathbb{P}^{2}\right)$.

Remark. We know that the infinite intersection of a countable collection of convergence sets is not necessarily a convergence set (see [21, Proposition 4.15]). We do not know whether the union of a countable collection of convergence sets is necessarily a convergence set. However, by Theorem 6.2.4, we have the following

Corollary 6.2.5. The union of a countable collection of convergence sets on $\Gamma$ is a convergence set on $\Gamma$. 


\section{CHAPTER 7}

\section{EXAMPLES}

In this chapter we give some examples of convergence sets or non-convergence sets. Recall that $\iota: \mathbb{C}^{n} \rightarrow \mathbb{P}^{n}$ is the embedding map defined by $\iota\left(z_{1}, \cdots, z_{n}\right)=\left[1: z_{1}: \cdots: z_{n}\right]$ and that $\Gamma$ is the image under the embedding $\mathbb{C}^{2} \rightarrow \mathbb{P}^{2}$ of the graph of a transcendental entire holomorphic function $\psi$. Then for $F \subset \mathbb{C}^{2} \subset \mathbb{P}^{2}$ and for $S \subset \mathbb{C}$,

$$
\iota(F)=\left\{\left[1: z_{1}: z_{2}\right] \in \mathbb{P}^{2}:\left(z_{1}, z_{2}\right) \in F\right\}, \quad \text { and } \Gamma(S)=\left\{[1: z: \psi(z)] \in \mathbb{P}^{2}: z \in S\right\} .
$$

Example 7.1. Let $E$ be the set of all "rational points" in $\mathbb{P}^{n}$. Then $E$ is a convergence set by Theorem 5.1.5.

Example 7.2. Let $F:=\left\{\left(z, e^{z}\right): z \in \mathbb{C}\right\} \subset \mathbb{C}^{2} \subset \mathbb{P}^{2}$. Then $\iota(F)=\Gamma(\mathbb{C})=\cup \Gamma\left(\Delta_{j}\right)$ when $\psi(z)=e^{z}$, where $\Delta_{j}=\{z \in \mathbb{C}:|z| \leq j\}$. Since $\Gamma(\mathbb{C})$ is a $G_{\delta}$ set in $\mathbb{P}^{2}$ and since each $\Gamma\left(\Delta_{j}\right)$ is projectively convex, we see that $\Gamma(\mathbb{C}) \in \operatorname{Conv}\left(\mathbb{P}^{2}\right)$ by Theorem 6.2.4. It follows that $\iota(F)$ is a convergence set in $\mathbb{P}^{2}$, and hence $F$ is a convergence set in $\mathbb{C}^{2}$.

Example 7.3. Let $F:=\{(z, 0): z \in \mathbb{C}\} \subset \mathbb{C}^{2} \subset \mathbb{P}^{2}$. Then $F$ is a convergence set in $\mathbb{C}^{2}$ since $\iota(F)=U_{0} \cap H_{2}$, where $H_{2}=\left\{\left[Z_{0}: Z_{1}: Z_{2}\right]: Z_{2}=0\right\}$ is a convergence set in $\mathbb{P}^{2}$. To see that $F$ is not a convergence set in $\mathbb{P}^{2}$, we seek for a contradiction. If $F$ is a convergence set in $\mathbb{P}^{2}$, by Proposition 6.1.6, $H_{2} \cap \iota(F)=\iota(F)$ is polar in $H_{2}$, a contradiction.

Example 7.4. Let $F:=\left\{\left(z, e^{z}\right):|z|=1\right\} \subset \mathbb{C}^{2} \subset \mathbb{P}^{2}$. Then $F$ is a convergence set in $\mathbb{P}^{2}$. To see this, consider $\Gamma\left(S_{j}\right)$ when $\psi(z)=e^{z}$ with $S_{j}=\left\{e^{i t}: 0 \leq t \leq 2 \pi-1 / j\right\}$. Then $\iota(F)=\cup \Gamma\left(S_{j}\right)$. Since $\iota(F)$ is $G_{\delta}$ and since each $\Gamma\left(S_{j}\right)$ is projectively convex, we see that $\iota(F)$ is a convergence set by Theorem 6.2.4.

Example 7.5. Let $S$ be the subset of $\mathbb{C}$ obtained by removing an open triangle from a closed triangle. Then $\Gamma(S)$ is a convergence set. This follows from an argument very similar to the previous example. 
Example 7.6. Let $S$ be the subset of $\mathbb{C}$ obtained by removing a finite number of open triangles from a closed triangle. Then $\Gamma(S)$ is a convergence set. This follows from the previous example and Proposition 5.1.3.

Let $\Lambda$ be a closed triangle in $\mathbb{C}$. The "open middle triangle" of $\Lambda$ is the open triangle whose vertices are the midpoints of the sides of $\Lambda$. Let $V_{1}$ be the open middle triangle of $\Lambda$. The set $\Lambda \backslash V_{1}$ is the union of three congruent closed triangles. Let $V_{2}, V_{3}, V_{4}$ denote the open middle triangles of the three closed triangles whose union is $\Lambda \backslash V_{1}$. Similarly, let $V_{5}, \ldots, V_{13}$ denote the open middle triangles of the nine closed triangles whose union is $\Lambda \backslash \cup_{j=1}^{4} V_{j}$. Continuing in this way, we obtain a sequence $\left\{V_{j}\right\}$ of open triangles. The set $F:=\Lambda \backslash \cup_{j=1}^{\infty} V_{j}$ is called Sierpiński's triangle (see picture). Let $F_{k}=\Lambda \backslash \cup_{j=1}^{k} V_{j}$ for $k=1,2, \ldots$ By Example 7.6, each $\Gamma\left(F_{k}\right)$ is a convergence set in $\mathbb{P}^{2}$. Note that $\Gamma(F)=\cap_{k=1}^{\infty} \Gamma\left(F_{k}\right)$.

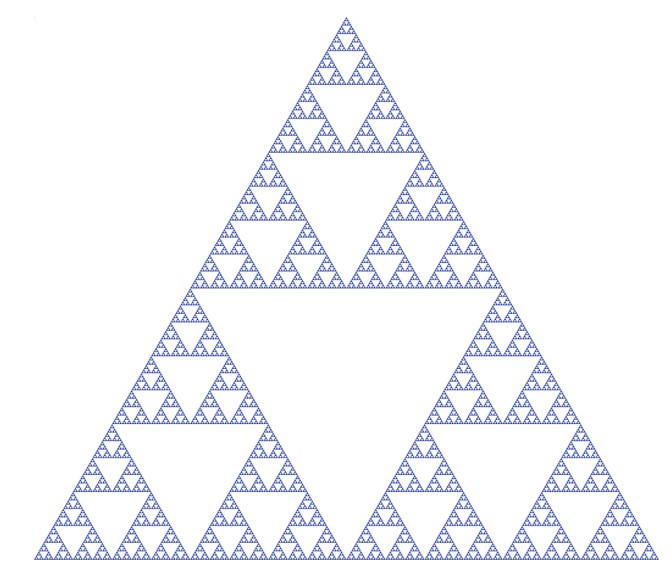

Figure 7.1: Sierpiński Triangle

Example 7.7. Let $F$ be the Sierpiński's triangle. Then $\Gamma(F)$ is not a convergence set. To see this, we seek for a contradiction. Suppose that $\Gamma(F)$ is a convergence set of $f=\sum p_{m}$ where $p_{m} \in \mathscr{H}_{m}\left(\mathbb{C}^{3}\right)$. Then $F=\cup S_{k}$, where $S_{k}=\left\{z \in \mathbb{C}:\left|p_{m}(1, z, \varphi(z))\right|^{1 / m} \leq k \quad \forall m\right\}$. Note that each $S_{k}$ is a closed subset of $\mathbb{C}$. By the maximum principle $\mathbb{C} \backslash S_{k}$ is connected for each $k$, which contradicts the Baire category theorem since $S_{k}$ is nowhere dense for each $k$ (see [21, Proposition 4.15]). 


\section{CHAPTER 8 CONCLUSION AND FUTURE WORK}

My primary goal of this dissertation is to introduce relative $\omega$-extremal functions, to study the relationship between $\omega$-plurisubharmonic functions and homogeneous polynomials, and to establish some results on pluripolar hulls and convergence sets. We expect to obtain some applications of convergence sets in dynamical systems presenting problems of small divisors. Also we believe $\omega$-plurisubharmonic functions will be a useful tool in the pluripotential theory of compact Kähler manifolds. We intend to study these in future work.

We now end this dissertation by raising several open problems.

Problem 8.1. Is a locally complete pluripolar set necessarily a complete pluripolar set? It was shown by Coltoiu (see [5]) that if a locally complete pluripolar set $F$ is closed, then $F$ is a complete pluripolar set.

Problem 8.2. Let $\left\{E_{j}\right\}$ be an ascending sequence of subsets of $\mathbb{P}^{n}$ and let $E:=\cup_{j=1}^{\infty} E_{j}$. Is it true that $\lim _{j \rightarrow \infty} \sigma\left(Z, s, E_{j}\right)=\sigma(Z, s, E)$ ? Proposition 3.1 .9 implies that it is true for the case $n=1$.

Problem 8.3. Is the union of two convergence sets a convergence set? 
REFERENCES 


\section{LIST OF REFERENCES}

[1] S.S. Abhyankar, T.T. Moh, A reduction theorem for divergent power series, J. Reine Angew. Math., 241 (1970), 27-33.

[2] B. Al-Shutnawi et al, On convergence sets of power series with holomorphic coefficients, preprint, 2017, arXiv:1707.04054.

[3] E. Bedford, B.A. Taylor, Plurisubharmonic functions with logarithmic singularities, Ann. Inst. Fourier (Grenoble), 38 (1988), no. 4, 133-171.

[4] E. Bedford, B.A. Taylor, A new capacity for plurisubharmonic functions. Acta Math., 149 (1982), no. 1-2, 1-40.

[5] M. Coltoiu, Complete locally pluripolar sets. J. Reine Angew. Math., 412 (1990), 108112.

[6] J. Deny, Sur les infinis d'un potentiel, C. R. Acad. Sci. Paris Sér. I Math., 224 (1947), $524-525$.

[7] A. Ediganian, J. Wiegerinck. The pluripolar hull ofthe graph of a holomorphic function with polar singularities, Indian Univ. Math. J., 52 (2003), no. 6 1663-1680.

[8] A. Ediganian, J. Wiegerinck. Determination of the pluripolar hull of graphs of certain holomorphic functions, Ann. Inst. Fourier, 54 (2004), no. 6, 2085-2104.

[9] T. Edlund, B. Jöricke. The pluripolar hull of a graph and fine analytic continuation, Ark. Mat., 44 (2006), no. 1, 39-60.

[10] P. Griffiths, J. Harris, Principles of algebraic geometry, John Wiley \& Sons, New York, 1978.

[11] V. Guedj, A. Zeriahi, Intrinsic capacities on compact Kähler manifolds, J. Geom. Anal., 15 (2005), 607-639.

[12] F. Hartogs, Zur Theorie der analytischen Funktionen mehrerer unabhängiger Veränderlichen, Math. Ann. 62 (1906), 1-88.

[13] F.R. Harvey, B. Lawson, Projective hulls and projective Gelfand transform, Asian J. Math., 10 (2006) 607-646.

[14] L. Hörmander, An Introduction to Complex Analysis in Several Variables, NorthHolland, Amsterdam, 1973. 


\section{LIST OF REFERENCES (continued)}

[15] B. Josefson, On the equivalence between locally polar and globally polar sets for plurisubharmonic functions on $\mathbb{C}^{n}$, Arkiv för Mat., 16 (1978), 109-115.

[16] M. Klimek, Pluripotential theory, Clarendon Press, New York, 1991.

[17] P. Lelong, On a problem of M.A. Zorn, Proc. Amer. Math. Soc., 2 (1951), 11-19.

[18] P. Lelong, Fonctions entières de typr exponentiel dans $\mathbb{C}^{n}$, Annales de l'Institut Fourier(Grenoble), 16 (1966), 271-318.

[19] N. Levenberg, R.E. Molzon, Convergence sets of a formal power series, Math. Z., 197 (1988), 411-420.

[20] N. Levenberg, E. Poletsky, Pluripolar hulls, Michigan Math. J., 46 (1999), no. 1, 151162.

[21] D. Ma, T.S. Neelon, On convergence sets of formal power series, J. Complex Analysis and its Synergies, (2015), 1:4, DOI 10.1186/s40627-015-0004-4.

[22] R. Pérez-Marco, A note on holomorphic extensions, preprint, 2000, arXiv:math/0009031.

[23] T. Ransford, Potential theory in the complex plane, Cambridge University Press, New York, 1995.

[24] J. Ribón, Holomorphic extensions of formal objects, Ann. Scuola Norm. Sup. Pisa Cl. Sci. (5), 3 (2004), 657-680.

[25] A. Sadullaev, An estimate for polynomials on analytic sets, Math. USSR Izvestia, 20 (1983), 493-502.

[26] A. Sadullaev, Plurisubharmonic measures and capacities on complex manifolds, Uspekhi Mat. Nauk, 36 (1981), 53-105; translation in Russian Math. Surveys 36 (1981), 61-119.

[27] A. Sathaye, Convergence sets of divergent power series, J. Reine Angew. Math., 283 (1976), 86-98.

[28] N. Sibony, Sur la frontière de Shilov des domaines de $\mathbb{C}^{n}$, Math. Ann. 273 (1985), $115-121$.

[29] J. Wiegerinck, The pluripolar hull of $\left\{w=e^{-1 / z}\right\}$, Ark. Mat, 38 (2000), no. 1, 201-208. 


\section{LIST OF REFERENCES (continued)}

[30] J. Wiegerinck, Pluripolar sets: hulls and completeness, Actes des Rencontres dAnalyseComplexe (Poitiers-Futuroscope, 1999), Atlantique, Poitiers, 2002, 209-219.

[31] J. Siciak, Extremal phurisubharmonic functions and capacities in $\mathbb{C}^{n}$, Sophia Kokyuroku Math., 14 (1982), Sophia University, Tokyo.

[32] A. Zeriahi, Ensembles pluripolaires exceptionnels pour la croissance partielle des fonctions holomorphes, Ann. Polon. Math., 50 (1989), 81-91. 